The Jackson Laboratory

The Mouseion at the JAXlibrary

Faculty Research 2021

Faculty Research

$1-15-2021$

Whole-genome screen identifies diverse pathways that negatively regulate ciliogenesis.

Marion Failler

Ariadna Giro-Perafita

Mikito Owa

Shalini Srivastava

Chi Yun

See next page for additional authors

Follow this and additional works at: https://mouseion.jax.org/stfb2021

Part of the Life Sciences Commons, and the Medicine and Health Sciences Commons 


\section{Authors}

Marion Failler, Ariadna Giro-Perafita, Mikito Owa, Shalini Srivastava, Chi Yun, David J Kahler, Derya Unutmaz, Francisco J Esteva, Irma Sánchez, and Brian D Dynlacht 


\title{
Whole-genome screen identifies diverse pathways that negatively regulate ciliogenesis
}

\author{
Marion Failler ${ }^{a}{ }^{\dagger}$, Ariadna Giro-Perafitaa, ${ }^{a}$, Mikito Owa ${ }^{a}$, Shalini Srivastavaa, Chi Yun ${ }^{a}$, \\ David J. Kahlera, Derya Unutmaz ${ }^{b}$, Francisco J. Esteva ${ }^{a}$, Irma Sánchez ${ }^{a, *}$, and Brian D. Dynlacht ${ }^{a, *}$ \\ aDepartment of Pathology and Perlmutter Cancer Center, New York University School of Medicine, New York, \\ NY 10016; bJackson Laboratory for Genomic Medicine and University of Connecticut School of Medicine, Farmington, \\ CT 06031
}

\begin{abstract}
We performed a high-throughput whole-genome RNAi screen to identify novel inhibitors of ciliogenesis in normal and basal breast cancer cells. Our screen uncovered a previously undisclosed, extensive network of genes linking integrin signaling and cellular adhesion to the extracellular matrix (ECM) with inhibition of ciliation in both normal and cancer cells. Surprisingly, a cohort of genes encoding ECM proteins was also identified. We characterized several ciliation inhibitory genes and showed that their silencing was accompanied by altered cytoskeletal organization and induction of ciliation, which restricts cell growth and migration in normal and breast cancer cells. Conversely, supplying an integrin ligand, vitronectin, to the ECM rescued the enhanced ciliation observed on silencing this gene. Aberrant ciliation could also be suppressed through hyperactivation of the YAP/TAZ pathway, indicating a potential mechanistic basis for our findings. Our findings suggest an unanticipated reciprocal relationship between ciliation and cellular adhesion to the ECM and provide a resource that could vastly expand our understanding of controls involving "outside-in" and "inside-out" signaling that restrain cilium assembly.
\end{abstract}

\author{
Monitoring Editor \\ Mark Ginsberg \\ University of California, \\ San Diego
}

Received: Feb 12, 2020

Revised: Nov 9, 2020

Accepted: Nov 12, 2020

\section{INTRODUCTION}

The primary cilium serves as a cellular "antenna" that protrudes from the cell surface of most quiescent mammalian cells, sensing and responding to the extracellular environment. Cilia act as con-

This article was published online ahead of print in MBoC in Press (http://www molbiolcell.org/cgi/doi/10.1091/mbc.E20-02-0111) on November 18, 2020.

TThese authors contributed equally.

*Address correspondence to: Brian D. Dynlacht (brian.dynlacht@nyumc.org); Irma Sánchez (irma.sanchez@nyumc.org).

Abbreviations used: BA, basal-like; BAIAP2, BAl1-associated protein 2; BSA, bovine serum albumin; ECM, extracellular matrix; EDA, ectodysplasin A; FA, focal adhesion; FAK, focal adhesion kinase; FBS, fetal bovine serum; FNDC3A, fibronectin type III domain protein; GAP, GTPase-activating protein; GEF, guanine nucleotide exchange factor; HER2, HER2-enriched; Hh, Sonic hedgehog; ITGB1, $\beta 1$ integrin; LA, Luminal A; LAMB4, laminin 4; LB, Luminal B; MAD, median absolute deviation; MTP, metalloproteinase; NL, normal-like; PBS, phosphate-buffered saline; PDE4D, phosphodiesterase 4D; PRG4, proteoglycan; qPCR, quantitative PCR; ROCK, Rho-associated protein kinase; shRNA, short hairpin RNA; SSMD, strictly standardized mean difference; TLN1, Talin-1; TNBC, triple-negative breast cancers; TNN, tenascin; VTN, vitronectin.

(C) 2021 Failler et al. This article is distributed by The American Society for Cell Biology under license from the author(s). Two months after publication it is available to the public under an Attribution-Noncommercial-Share Alike 3.0 Unported Creative Commons License (http://creativecommons.org/licenses/by-nc-sa/3.0).

"ASCB®," "The American Society for Cell Biology $\bigotimes^{\prime}$, and "Molecular Biology of

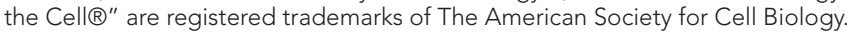

duits for signaling through Sonic hedgehog $(\mathrm{Hh})$, Notch, Wnt, and other pathways essential for the regulation of a number of biological processes, including cell polarity, migration, and differentiation (Goetz and Anderson, 2010; Sánchez and Dynlacht, 2016). Defects in the assembly, structure, or function of the primary cilium are linked to a broad spectrum of diseases, collectively known as ciliopathies, and these pathologies are associated with a plethora of developmental defects (Wang and Dynlacht, 2018). Emerging evidence suggests that some types of cancers could constitute a novel class of ciliopathies, as several studies have demonstrated that ciliation of epithelial cells is sharply decreased in cancers, including breast, prostate, and pancreatic tumors, as compared with normal tissue (Wang and Dynlacht, 2018).

Loss of cilia appears to be an early event in the genesis of some types of tumors, as neoplasia that anticipate the appearance of pancreatic and breast adenocarcinomas exhibit near-complete loss of this organelle (reviewed in Sánchez and Dynlacht, 2016). Taken together with the observation that persistent ciliation could abolish the ability of cells to form a mitotic spindle, these studies suggest that primary cilia may have tumor-suppressive functions in these types of cancers (Mans et al., 2008; Menzl et al., 2014). In contrast, several studies have shown that in some tumors which are driven by 
Hedgehog pathway activation, cilia are protumorigenic (Sarkisian and Semple-Rowland, 2019). Thus, while much information has been obtained over the past decade regarding the process of cilium assembly, mechanistic insights into ciliary disassembly-which is normally instigated on cell cycle entry-and cilia loss or maintenance during tumorigenesis are largely lacking. Indeed, the mechanisms regulating the suppression of ciliation during development and maturation in the adult remain largely unknown.

During mammary development, primary cilia are specifically required for appropriate branching morphogenesis, producing a bilayered ductal system composed of apically oriented luminal epithelial cells encircled by contractile and basement membraneassociated basal cells (McDermott et al., 2010). Primary cilia are found on both luminal and basal cells during branching morphogenesis, but they are largely absent from luminal cells after completion of breast development (Mans et al., 2008). Thus, it is likely that primary cilia play important roles in the biology of normal basal breast cells. In support of this conclusion, several studies of normal breast tissue have revealed that the $\mathrm{Hh}$ ligand is secreted by the luminal cells of the breast, whereas Hh-responsive cells are enriched in the basal layer and the associated stroma (O'Toole et al., 2011; García-Zaragoza et al., 2012).

Breast cancer is the most frequently diagnosed malignant disease and a leading cause of cancer-related deaths in women worldwide. Breast cancers are classified into five major subtypes: Luminal A (LA), Luminal B (LB), HER2-enriched (HER2), basal-like (BL), and normal-like (NL) based on gene expression profiles (Sorlie et al., 2001). Triple-negative breast cancers (TNBC) lack the expression of estrogen, progesterone, and HER2 amplification and are primarily represented by the basal-like subtype. Gene expression analysis of TNBC tumors identified two major subtypes of TNBC, basal-like (or basal A) and Mesenchymal-like/Claudin-low (or basal B). TNBC have the worst prognosis of all breast tumor subtypes, as there is a lack of effective therapies. The basal B subtype of breast cancer is thought to retain cancer stem cell-like and mesenchymal-like expression profiles and is strongly associated with metastasis (Lehmann et al., 2011). Interestingly, primary cilia were identified in basal B subtype epithelial cell lines at a low frequency, as compared with normal breast epithelial cells and luminal cancer cells, but they were not observed in cells of the basal A subtype for reasons that remain unknown (Yuan et al., 2010). Primary cilia are lost in epithelial cells at a very early stage during breast cancer development and are present only in intermingled stromal cells, raising the possibility that their disappearance may be important for tumor development in breast cancer (Mans et al., 2008; Menzl et al., 2014). Therefore, our data support testing whether restoration of this organelle in epithelial cells of basal breast cancers could, in principle, restrain tumor development by rebalancing signaling pathways involved in cell proliferation that are deregulated in TNBC (Howe and Brown, 2004; O'Toole et al., 2011).

In an effort to uncover mechanisms through which breast cancers block ciliation, we performed a whole-genome siRNA screen to identify inhibitors of ciliogenesis in normal and basal breast cancer cells. Unexpectedly, we identified a cohort of genes whose removal promotes ciliation in normal and cancer cells through unanticipated mechanisms, and we focused in particular on a group of proteins associated with the extracellular matrix (ECM) and integrin-mediated cellular adhesion. Our results strongly suggest that certain cancer cells, like their normal counterparts, harness aspects of cellular adhesion to suppress ciliation. Our studies provide a valuable resource for further exploration of novel pathways that restrain cilium assembly in normal and pathological states.

\section{RESULTS AND DISCUSSION Whole-genome screen for suppressors of ciliation in breast cancer}

An important outstanding question concerns the extent to which cancer cells retain the ability to ciliate, since this decision alters signaling events and proliferation. Hs578T basal B breast cancer cells, derived from a TNBC patient, retain the ability to assemble cilia at low frequency (Yuan et al., 2010), suggesting that suppression of inhibitory proteins might unleash their potential to ciliate robustly in the presence or absence of mitogenic stimuli. Indeed, treatment of these cells and other basal B breast cancer cells with cytochalasin D to depolymerize actin or transfection of siRNAs that target a known inhibitor of ciliogenesis (NEK2/KIF24) provoked a marked enhancement of ciliation in the presence of mitogens (Kim et al., 2015b, and unpublished data). In addition, serum deprivation enhanced ciliation in these cells, although the frequency of cilium assembly was considerably dampened in comparison with normal epithelial cells (see below).

To comprehensively discover novel genes and pathways that promote loss of cilia and enhanced proliferation in breast cancer, we performed a high content RNAi screen in Hs578T cells (Figure 1). Although whole-genome siRNA screens have been used to identify genes essential for cilia formation, it is considerably more challenging to study the disassembly of this critical organelle, and relatively few inhibitors of ciliogenesis have been identified. We took advantage of Hs578T cells for several reasons. First, this cell line exhibits the capacity to ciliate at low levels (even in the face of increased cell density and serum deprivation), enabling us to expand the dynamic range of ciliation events after gene silencing and thereby increasing the sensitivity of our assay. Second, we hoped that our screen could provide a "toolkit" to explore the compendium of genes whose inhibition could allow reciliation in cancer cells. About 22,000 genes representing the entire human genome were individually silenced in triplicate with the siRNA library to allow cilium assembly. The siRNA library was comprised of pools of three individual siRNAs targeting each gene.

We assayed for assembly of primary cilia $96 \mathrm{~h}$ after reverse transfection by staining cells with DAPI (to determine cell number) and antibodies against the cilium marker, ARL13b, automated fluorescent imaging, and identification of cilia using a spot detector algorithm (Figure 1) followed by visual inspection. Statistical significance for differences in the number of cells and cilia was calculated using a robust $z$ score (see Materials and Methods; Zhang, 2011). Potential hits were identified by filtering out pools that provoked cell death or immediate growth arrest (which could itself increase ciliation) and using a stringent cutoff calculated from the ratio of ciliated cells/total cell number (Rob Z score $>3$ and strictly standardized mean difference $(\mathrm{SSMD})>$ 1.645; Supplemental Table S1) for at least two replicates (see Materials and Methods). For batches with a lower overall ciliation efficiency, the cutoffs were adjusted to the median and the median absolute deviation (MAD) of robust $z$ scores of the positive controls (med + 1.45*MAD > Zcilia cutoff). Among the candidates that surpassed these thresholds, we identified 356 genes $(\sim 1.6 \%$ of the genome) for which knockdown increased the number of ciliated cells without affecting cell survival (Figure 1; Supplemental Table S1). Gene Ontology analysis indicated that the list of candidates was enriched in unanticipated clusters of genes pertaining to control of mitochondrial biogenesis and function, cell cycle, protein translation, cell adhesion, migration, and signaling, among others (Table 1). These results suggest that, in cancer cells, metabolic state, protein translation, and cell adhesion are linked to cilia loss.

Next, we performed a confirmatory screen using Hs578T and four other cell lines, including two normal cell lines that robustly 

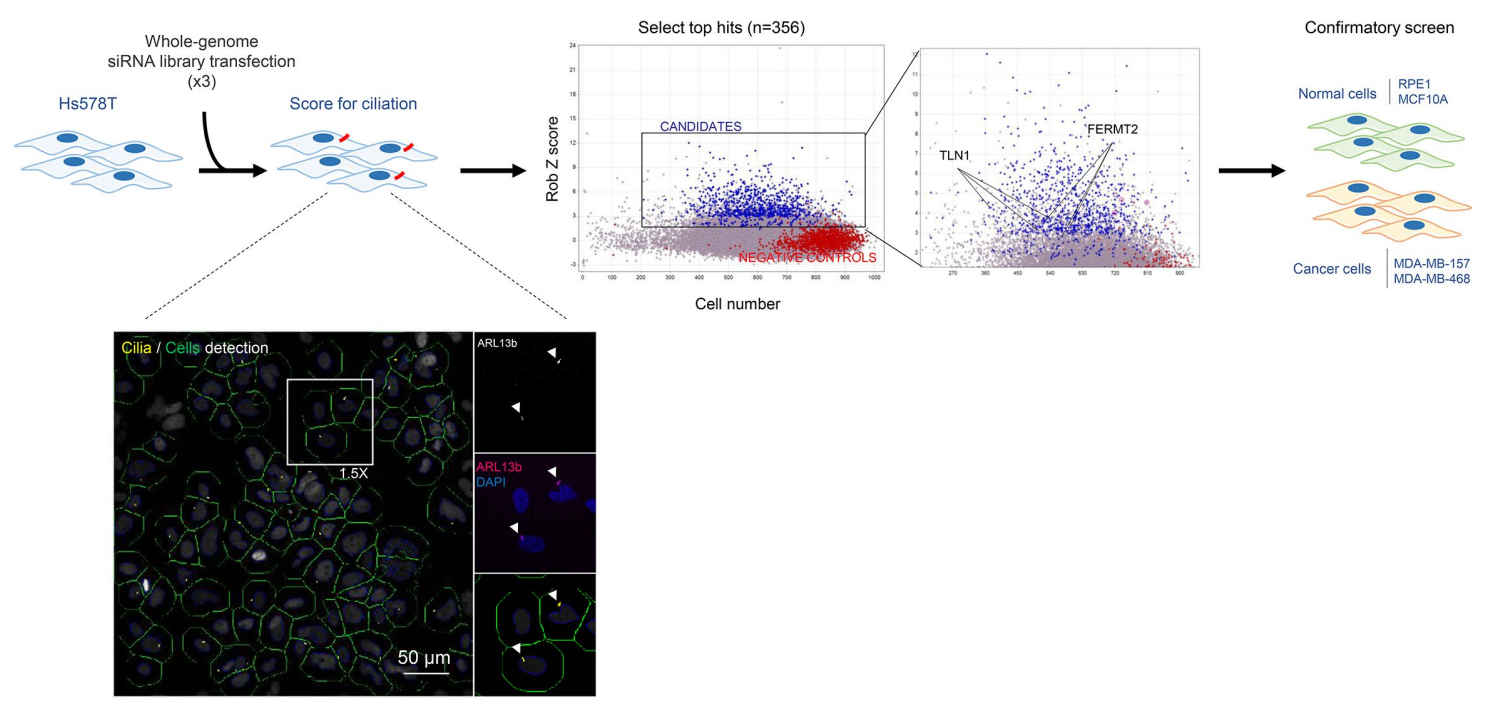

FIGURE 1: Genomewide siRNA screen identifies ciliation inhibitors. Schematic indicating overview of our siRNA screening strategy and approach. Representative high content imaging of cilia (Arl13b, pink) and nuclei (DAPI, blue) in $\mathrm{Hs578T}$ cells treated with a pool of three siRNAs targeting each gene. Software identified cilia (yellow) in a validated region (green line) around the nuclei (blue). Scatter plot showing the Rob Z score corresponding to the extent of ciliation vs. cell number for all siRNA library replicates (gray and blue dots) and nonspecific siRNAs (negative control, red dots). Higher magnification of candidate siRNA (blue dots) that do not affect cell survival (cell number cutoff $=180$ ) and have a zcilia cutoff $>3$ or Med Rob Z ctr $+1.45^{\star}$ MAD defined for individual batches. Arrows show results for two candidate genes. All candidates were subsequently tested in a confirmatory screen on normal and breast cancer cell lines. Scale bar $=25 \mu \mathrm{m}$.

ciliate (RPE1, retinal pigmented epithelial cells, and MCF-10A, nontumorigenic breast epithelial cells), a second basal B breast cancer cell line (MDA-MB-157), and a basal A breast cancer cell line that has essentially lost its ability to ciliate altogether (MDA-MB-468). We verified that the vast majority of identified targets survived the secondary screen in Hs578T (79\%) and both normal cell lines (94\% in aggregate). Interestingly, there was a significant overlap between hits in Hs578T and MDA-MB-157 (46\%), but relatively few hits were shared with MDA-MB-468 (18\%) (Figure 2A). These results confirm the specificity of our screen and the ability to reciliate basal $B$, but not basal A, subtype breast cancer cells, and they suggest that either the differentiation state of cells derived from the latter tumor type, the presence of additional genetic alterations, or other yet-tobe-defined differences prevent efficient cilium assembly. In addition, since expression levels of our candidates can vary significantly across individual cell lines, this could, in part, explain cell type-specific differences. Notably, our list of candidate genes, with the exception of one hit (PRC1), did not overlap with hits from a prior high-throughput screen (Kim et al., 2010) designed to identify regulators of ciliogenesis in RPE1 cells or a screen in mouse inner medullary collecting duct (IMCD3) cells focusing on genes needed for ciliogenesis (Wheway et al., 2015) (Supplemental Table S2). Moreover, we note that both prior screens, carried out using vastly different siRNA libraries and experimental conditions, largely identified positive regulators of ciliogenesis, further reinforcing the novelty of our findings. Importantly, our studies suggested that basal B tumor cells can be reciliated through diverse mechanisms via gene silencing. In all subsequent studies, we focused exclusively on a subset of hits that passed our confirmatory screen.

\section{Identification of novel classes of ciliogenesis inhibitors}

Cytoskeletal proteins. Several lines of evidence suggested that our screen was successful. First, $\sim 15 \%$ of the candidates are known to be linked to cilium/centrosome biogenesis or function (Figure 2B; genes in bold in Table 1), and the majority of them are listed in centrosomeDB (http://centrosome.cnb.csic.es/human/centrosome), SYSCilia (http://www.syscilia.org/goldstandard.shtml), or CiliaCarta databases. Among these candidates, TTK/Mps1, Agbl5/CCP5, Cep128, and DYNC112 have been characterized as negative regulators of ciliogenesis (Majumder and Fisk, 2013; Gupta et al., 2015; Inaba et al., 2016; He et al., 2018; Hong et al., 2018). Second, two major components of the cytoskeleton, microtubules and microfilaments, have been previously implicated in the suppression of ciliogenesis. STMN3 was uncovered in our screen, suggesting that its function in destabilizing microtubules could initiate ciliogenesis, like its paralog, STMN1 (Pitaval et al., 2017). PARVA, a component of the focal adhesion (FA) complex regulating actin cytoskeletal dynamics and cell signaling, was shown to regulate cilium length (Kim et al., 2010), and our identification of its paralog, PARVB, likewise links this protein — with roles in adhesion, spreading, and motility - to the regulation of ciliogenesis.

Moreover, we identified several genes involved in promoting assembly of the actin network, including gamma-actin (ACTG1), BAIAP2/IRSp53, and WASF2 (Figure 2B; Table 1). Consistently, a previous high-throughput screen demonstrated that actin network dynamics contribute substantially to ciliogenesis and cilia length and indicated that assembly of the actin cytoskeleton negatively regulates ciliogenesis (Kim et al., 2010). Many significant gaps remain, however, in our understanding of actin-based regulation of ciliation. Among the different forms of filamentous or F-actin are branched F-actin and stress fibers. Branched F-actin is nucleated by the Arp $2 / 3$ complex and is primarily enriched near the cell cortex, where it contributes to the formation of the lamellipodium, membrane protrusions at the leading edge of migrating cells. Arp2/3 complex activation is positively regulated by signaling pathways that involve Rho-family GTPases, including CDC42 and Rac1. Rac1 binds to one of our hits, BAl1-associated protein 2 (BAIAP2), which, in turn, associates with another hit, WASF2, stimulating Arp2/3 and 


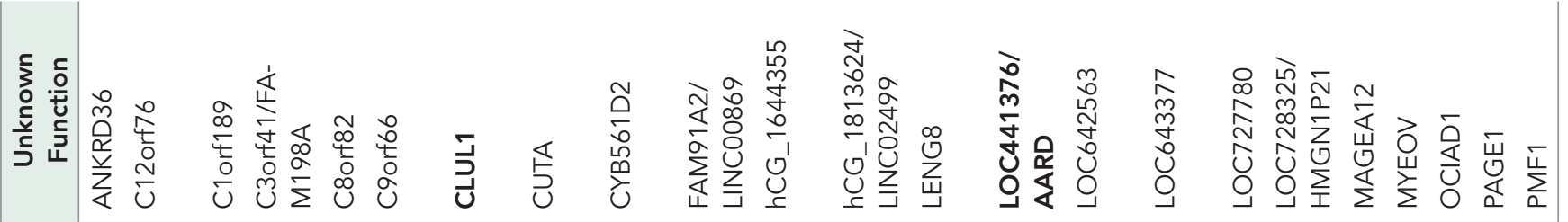

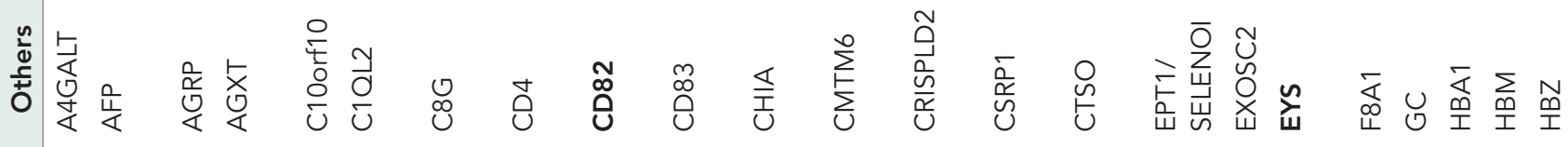

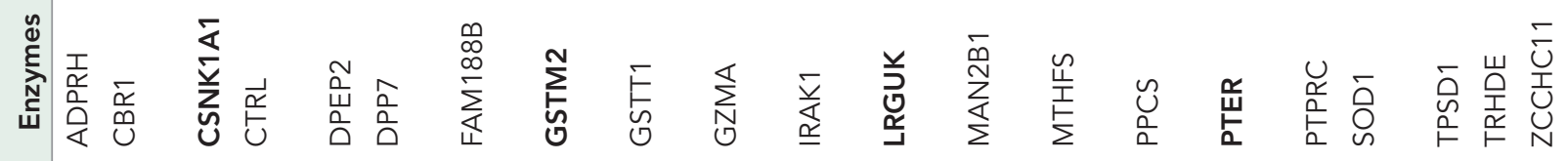

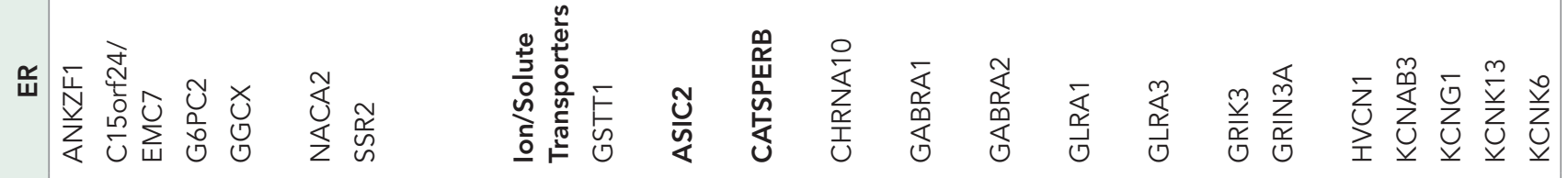

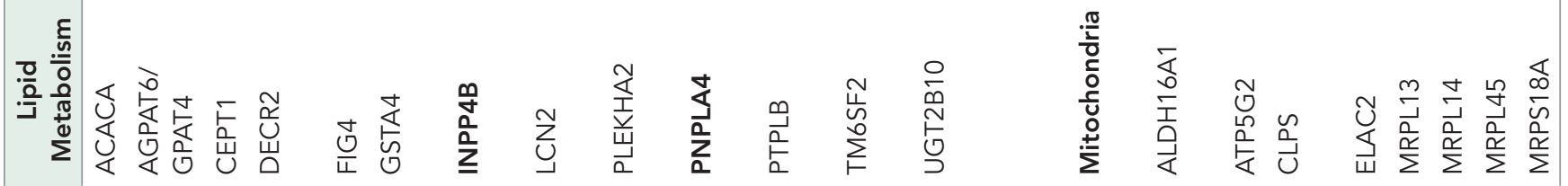

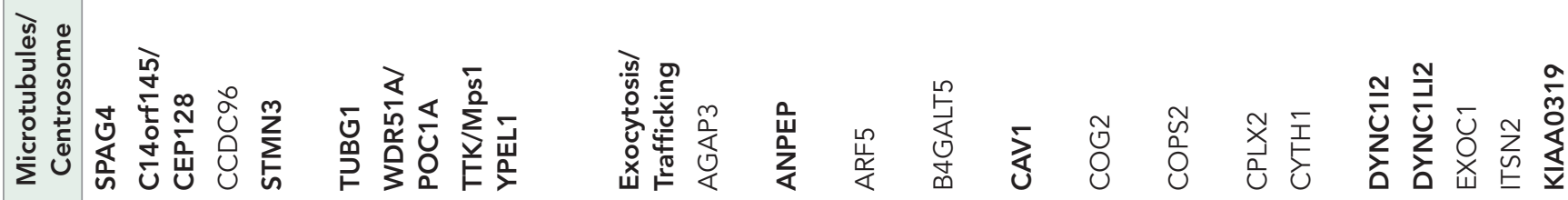

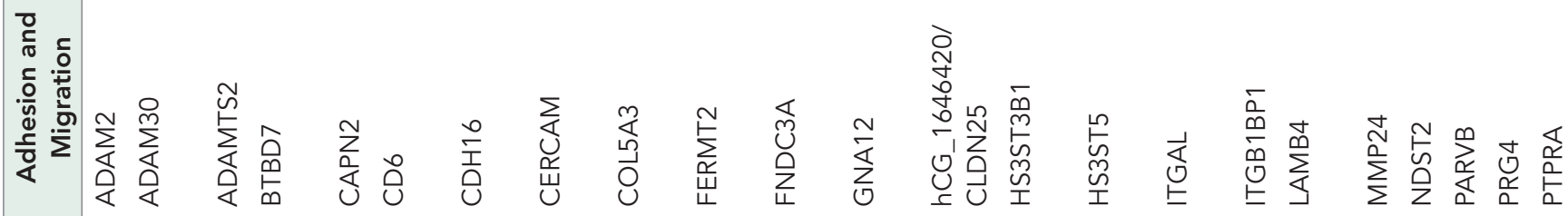

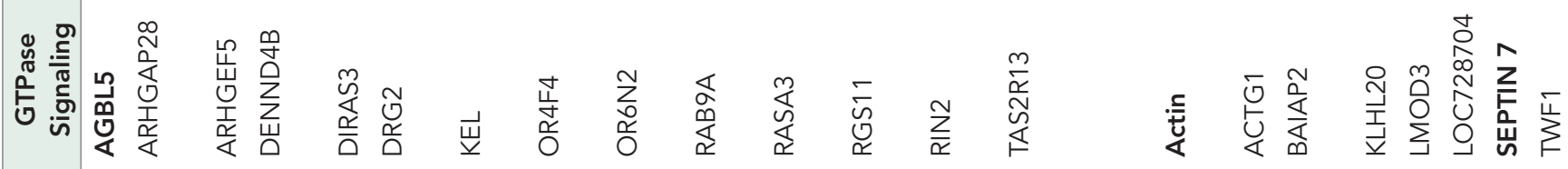

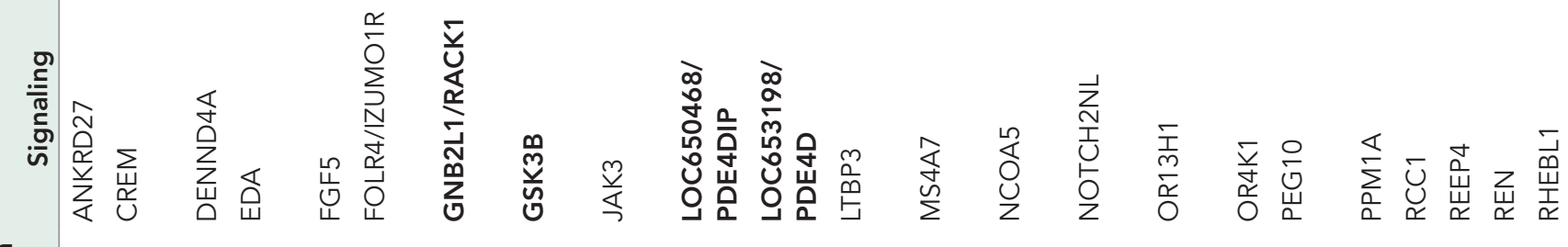

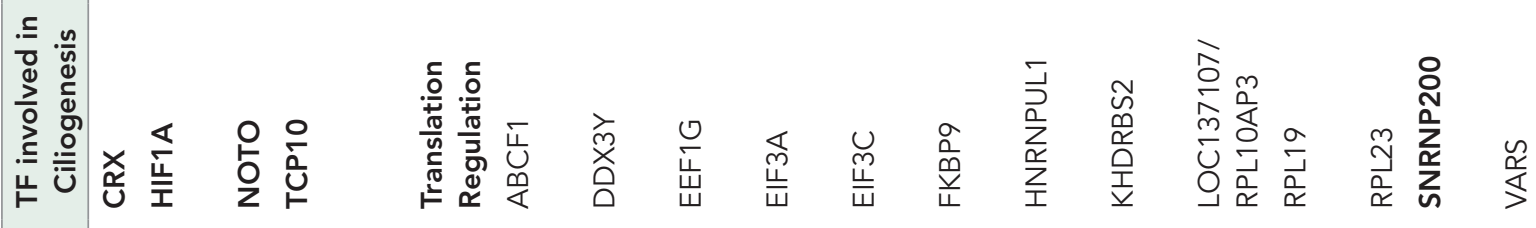

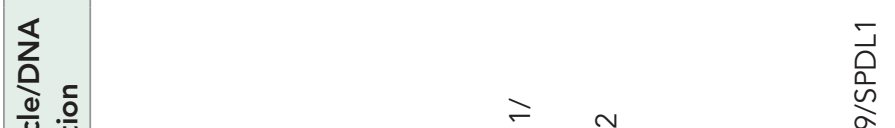

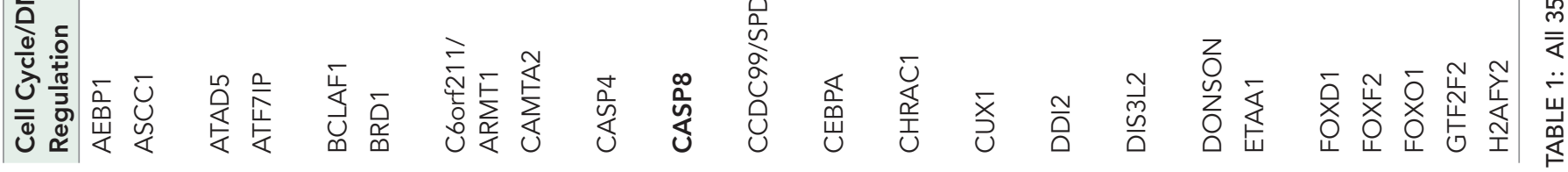




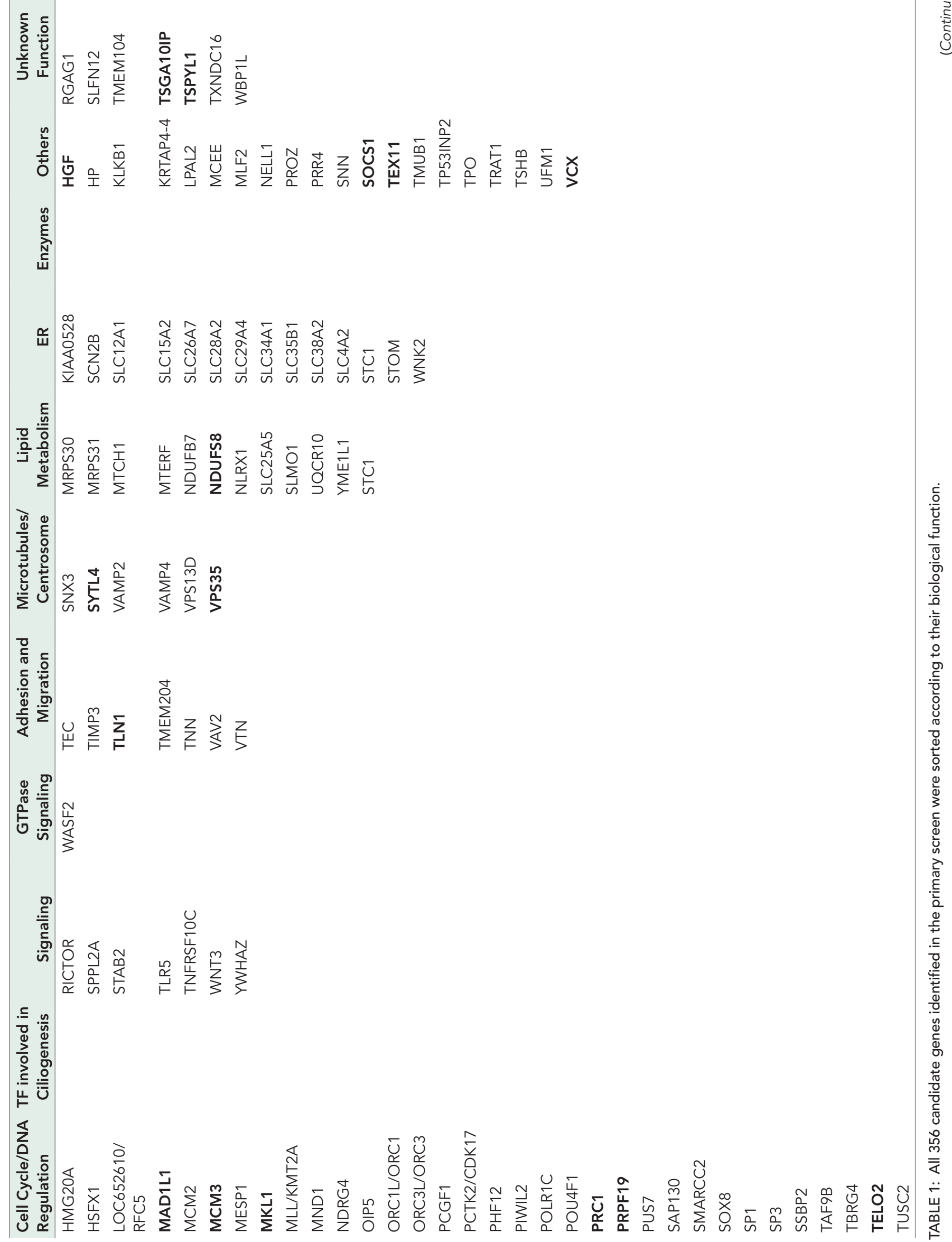




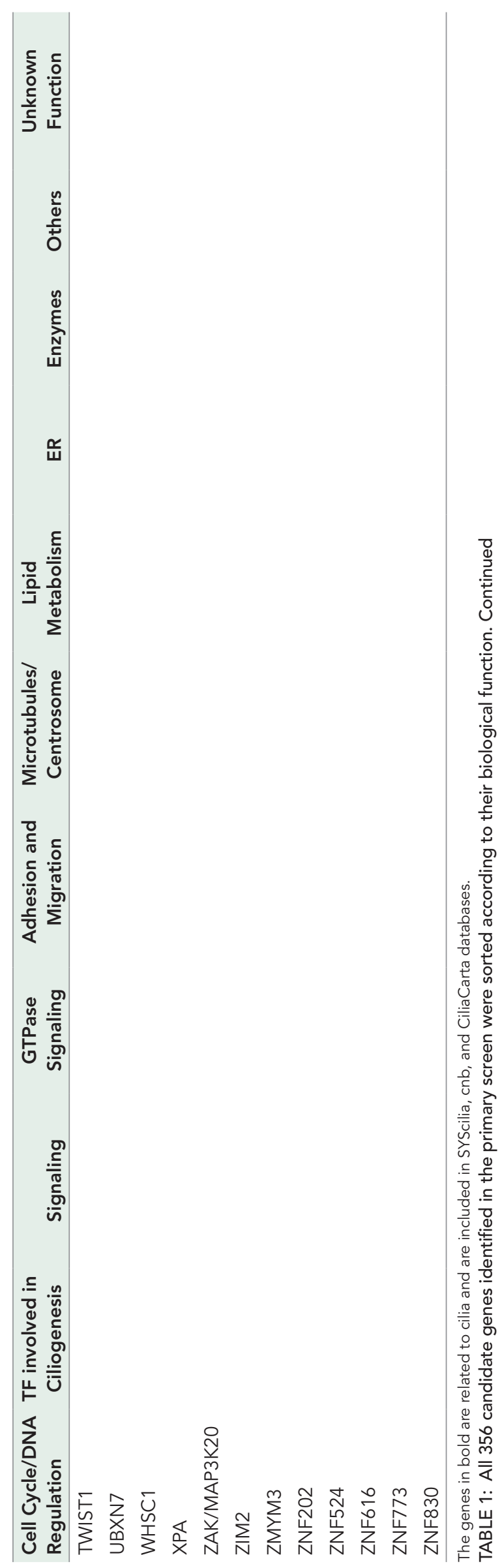

leading to actin polymerization (Miki et al., 2000). Assembly of branched actin networks also inhibits cilia formation by modulating membrane vesicle trafficking around centrosomes associated with the initial steps of ciliogenesis (Kim et al., 2010). Conversely, actin depolymerization promotes ciliary assembly and elongation, and inhibitors of actin polymerization, such as cytochalasin D, rapidly and potently stimulate primary cilia formation and aberrant elongation of cilia under conditions that normally promote ciliary disassembly, including serum stimulation of cultured cells (Kim et al., 2010). It is thought that cytochalasin D exerts these cilia-promoting effects at low concentrations incapable of affecting the integrity of stress fibers (Kim et al., 2010), implying that cilia formation is actuated through the inhibition of certain highly dynamic form(s) of F-actin. Recently, the apical membrane protein, Cav1, also identified in our screen, was shown to negatively regulate primary cilium length by modulating RhoA GTPase activity, another promoter of apical actin polymerization (Rangel et al., 2019). Similar to cytochalasin D action, the absence of Cav1 expression disrupted the apical actin meshwork, but not stress fibers, and increased the size of cilia.

Mitochondrial function. Surprisingly, about $\sim 6 \%$ of the identified hits were related to mitochondrial function (Figure 2B; Table 1). The role of mitochondria in the respiratory chain suggests that ATP generation may be important for cilium disassembly or regulation. Indeed, almost half of the candidates that we identified in this category are involved in respiratory chain function (e.g., ATP5G2, NDUFB7, NDUFS8, SLC25A5), while the remainder includes mitochondrial proteostasis and ribosome genes (YME1L1, MRPLs13, 14, and 45 and MRPS18A, 30, and 31), suggesting that mitochondrial genes contribute to ciliogenesis. Of note, while this article was in preparation, another group found that inhibiting mitochondrial function led to increased cilium length in human fibroblasts (Burkhalter et al., 2019). Moreover, exome sequencing identified human patient mutations in mitochondrial translation genes that were linked to OXPHOS diseases exhibiting heterotaxy, a feature of ciliopathies. Indeed, we identified a cohort of these genes in our screen, and a rare variant in SLC25A5 was identified by exome sequencing. While further studies are required to understand the exact links between mitochondrial and respiratory chain function and cilia formation, these results suggest that the function of a group of mitochrondrial genes can be linked to cilium dynamics and human disease.

Ion/solute transporters and channels. We also showed that $~ 8 \%$ of our hits were neurotransmitters, solute carriers, and ion channel proteins (Figure 2B; Table 1). Recently, drugs that target neurotransmitter function and ion channel modulators were shown to restore primary cilium expression in different cancer cells (Khan et al., 2016), probably by affecting levels of cAMP, calcium, or other ions that are established regulators of cilium formation and/or cilium length. Accordingly, the potassium channel, Kv10.1 (KCNH1), was shown to promote ciliary disassembly when overexpressed, and conversely, siRNA-mediated depletion of Kv10.1 resulted in aberrant ciliogenesis in proliferating cells (Sánchez et al., 2016). These studies reinforce the idea that strict control of ion and neurotransmitter concentrations in the cell is essential for timely cilia formation. Therefore, our unbiased identification of components of diverse potassium channels (including KCNG1, KCNK6, KCNK13, KCNAB3) pinpoint these proteins as inhibitors of ciliogenesis and suggest the potential utility of targeting them in cancer.

FAs, integrins, and ECM. Tumor invasion and metastasis correlate with altered cell-cell and/or cell-matrix adhesion properties, and 
A.

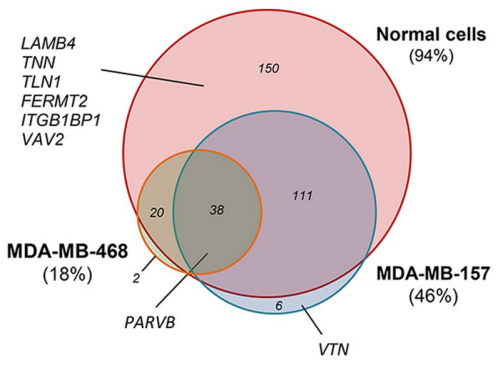

B.

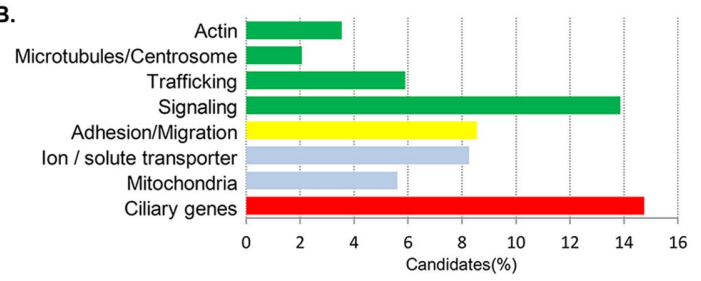

C.

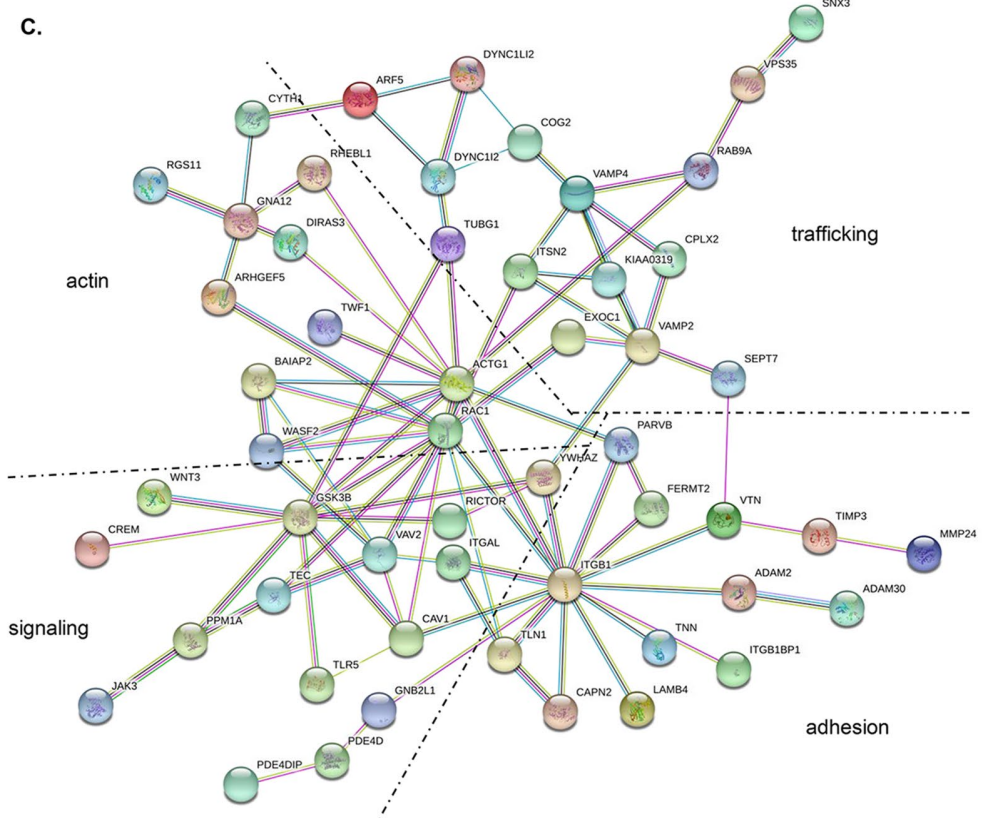

FIGURE 2: Confirmatory screen verifies novel negative regulators of ciliogenesis in multiple cell lines. (A) Venn diagram showing the number of candidate genes identified in Hs578T and shared by normal cells (MCF10A and RPE1, combined), a second basal B cell line (MDA-MB-157), and a basal A cell line (MDA-MB-468). (B) Bar graph showing the proportion of candidate genes involved in the indicated biological functions according to Gene Ontology categorization; $15 \%$ of the candidates are known to be linked to cilium and centrosome biogenesis or function (red). (C) STRING network analysis showing extensive connections between two hubs centered on ITGB1 and Rac1 and candidate genes involved in actin cytoskeleton, cell adhesion, trafficking, and signaling pathways identified in our screen.

the basal B subtype of breast cancer is strongly associated with metastasis. The latter is a multistep process dependent on integrindependent ECM adhesion complexes which directly communicate with the actin cytoskeleton, focal/nascent complexes, and FAs. Fo$\mathrm{cal} /$ nascent complexes reside near the leading edge of the cell, are short lived, and are not contractile. Focal complexes mature into larger structures termed FAs and can assemble large actin myosin filaments (stress fibers), which are highly contractile and are located away from the cell front, toward the center and rear of the cell. Cellular migration is accomplished in several steps, first by the anchoring of the cell front to the ECM through focal complexes, which connect to the intracellular actin network through filopodia and lamellopodia, followed by contraction of stress fibers which pull on the cell body, thereby retracting the trailing edge of the cell.

The Ras-related GTP-binding proteins, Rho, Rac, and Cdc42, each modulate the polymerization of actin to produce stress fibers, lamellipodia, and filopodia, respectively.

Activation of this family of proteins is mediated by guanine nucleotide exchange factors (GEFs) and inactivation by GTPase-activating proteins (GAPs). An additional level of GEF regulation is mediated by the $G$ protein alpha subunit of heterotrimeric $G$ proteins, which, when bound to GTP, interact with multiple Rho GEFs to regulate the actin cytoskeleton (Dhanasekaran and Dermott, 1996). Interestingly, our screen identified multiple, activating components of the Rho GEF-Rho axis, including the G protein alpha subunit, GNA12, a regulator of Rho GEFs, and two Rho GEFs, Vav2 and ARHGEF5, as suppressors of ciliation. Vav2 activates Rac1 and Cdc42 and is necessary for integrin-dependent activation of Rac1 during cell spreading (Marignani and Carpenter, 2001). ARHGEF5 is required for integrin-dependent activation of the Rho-associated protein kinase, ROCK. The latter regulates cell migration by promoting cellular contractility through the regulation of myosin light chain phosphorylation (Bhadriraju et al., 2007).

Interestingly, our screen also uncovered a negative regulator of cAMP production, phosphodiesterase 4D (PDE4D; LOC653198), depletion of which enhanced ciliation. PDE4D acts as a regulator of cancer cell invasion by interacting with FA kinase (FAK) through the receptor for activated C-kinase, RACK1/GNB2L1 (Delyon et al., 2017), which was also recovered in our screen and which interacts with actin to promote Rac1 activation. RACK1 also enables the interaction of PKC $\varepsilon$ with $\beta 1$ integrin (ITGB1) to promote tumor cell adhesion and migration (Besson et al., 2002). To examine the relationship between these proteins and ciliation, we blocked the activity of RACK1 and PDE4D using either individual short hairpin RNAs (shR$\mathrm{NAs}$ ) (targeting sequences distinct from those used in the screen) or a small molecule inhibitor of PDE4D (GEBR-7B). Interestingly, suppression of RACK1 or PDE4D protein expression or activity markedly enhanced ciliation of Hs578T cells (Supplemental Figure S1), independently confirming that these proteins inhibit ciliogenesis.

The identification of genes in our screen that suppress ciliation and that function as key regulators of integrin-dependent adhesion and migration prompted us to inspect more closely another cohort of proteins identified in our screen that are specifically involved in the stabilization and activation of integrins at FAs. Talin-1 (TLN1) and FERMT2/kindlin 2 are intracellular integrin-binding adaptor proteins essential for integrin activation (Kim et al., 2011), and both proteins were identified in our screen. Significantly, our screen uncovered multiple genes that regulate the binding of TLN1 to ITGB1, including CAPN2 (Yan et al., 2001) and FERMT2 (Li et al., 2017). FERMT2 expression is elevated in aggressive breast cancers with reduced patient survival and conversely, deficiency of this gene inhibits invasive and migratory properties in vitro (Sossey-Alaoui et al., 2017). 
CYTH1, another hit, is also able to activate integrins and regulate adhesion and engraftment (Rak et al., 2017).

Perhaps most remarkably, we identified an extensive network of ECM components in our screen, including vitronectin (VTN), tenascin (TNN), laminin, beta 4 (LAMB4), collagen (COL5A3), fibronectin type III domain protein (FNDC3A), and ectodysplasin A (EDA). Proteins in this group have been shown to play a role in cell proliferation, migration, and invasion and to enhance the metastatic potential of breast cancer cells through ITGB1. We also identified a proteoglycan (PRG4) and sulfotransferases (HS3ST5, HS3ST3B1, NDST2) that modify an ECM component, heparin sulfate, in normal and Hs578T cells, suggesting that ECM modifications can regulate cilia formation. Further, several genes identified in our screen (MMP24, ADAM2, ADAM30, ADAMTS2, and TIMP3; Table 1) regulate adhesion through their metalloproteinase (MTP) activity. It has been shown that MTPs promote tumor growth, invasion, and metastasis in breast cancer and are up-regulated in breast cancers with poor outcomes (Radisky and Radisky, 2015). The MTP inhibitor, TIMP3, also suppressed ciliogenesis in our screen. Thus, our work greatly expands on the number of metalloproteases with the potential to modulate ciliogenesis (Gopalakrishnan et al., 2012; Nandadasa et al., 2019), although further studies are required to identify the specific roles of these proteins in cilium assembly/disassembly. Interestingly, we note that although proteins conferring cell-ECM interactions were enriched in our screen, relatively few proteins mediating cell-cell adhesion were identified, thus implicating a specific type of cell adhesion, outside-in signaling, or both, in the negative regulation of cilium assembly and demonstrating, for the first time, pervasive links between the ECM and ciliogenesis.

Migration requires rapid FA turnover, with the assembly of FAs generally occurring at the front of the migrating cell, while disassembly occurs both at the leading edge, where it accompanies the formation of new protrusions, and at the cell rear where it promotes tail retraction (Supplemental Figure 2B). Cells with more stable FAs are generally less motile and thereby less invasive (Bijian et al., 2013). Several genes shown to be involved in FA and integrin turnover, including DYNC112, CAV1, ANPEP, VAMP2, Arf5, RIN2, and VAMP4, were identified in our screen, highlighting the importance of FA and integrin dynamics in the formation of cilia.

In addition to genes regulating the integrin network, we also identified Tec tyrosine kinase, which transduces integrin signaling to regulate the actin cytoskeleton (Gomez-Rodriguez et al., 2007), and PARVB, which interacts with $\alpha$-actinin and mediates integrin signaling and reorganization of F-actin induced by initial cell-substrate interactions (Yamaji et al., 2004).

\section{Extensive connections among FAs, integrins, and the actin network regulate cilium assembly}

Unexpectedly, the results of our screen suggested a broad and critical role for integrin $\beta 1$-mediated cell adhesion in ciliogenesis, as a substantial number of candidates identified in our screen are involved in cell-matrix adhesion, as well as migration (8.6\%) (Figure 2B). Links between the ECM and cilium function have been noted, but thus far the connections have remained indirect and enigmatic (McGlashan et al., 2006; Seeger-Nukpezah and Golemis, 2012; Xu et al., 2018). When we examined these hits with the functional protein-protein interaction network analysis software, STRING, we confirmed connections to multiple networks involving the actin cytoskeleton, ECM and adhesion, trafficking, and key signaling pathways (Supplemental Figure 2A). Limited studies have supported possible localization of integrins to cilia (Praetorius et al., 2004; Goodman and Zallocchi, 2017), but their functional role, if any, within this organelle has not been studied extensively. STRING analysis revealed that ITGB1 interacts with many hits from our screen, including genes involved in adhesion, signaling, and trafficking, that converge on Rac1 activation (Figure 2C). Rac1 is a key Rho GTPase controlling actin cytoskeleton organization, cell adhesion to the extracellular matrix, and migration. In addition to its role in lamellipodia formation, Rac1 is essential for actin stress fiber and FA assembly (Guo et al., 2006). Rac1 is overexpressed in breast tumors from the early stages of malignant transformation and throughout the metastatic process (Schnelzer et al., 2000). Indeed, when we manually inserted ITGB1 and Rac1 within our network of hits, we found that they acted as hubs to integrate nearly $60 \%$ of all candidates into a more robust, extended network (Figure $2 \mathrm{C}$ ). Thus, our screen uncovered an expansive network linking proteins within the cellular milieu to cell surface proteins that signal to Rac1 and the actin network, thereby convergently acting to suppress ciliation (summarized in Supplemental Figure 2B)

\section{Suppression of ECM and integrin signaling genes alters cell adhesion and promotes ciliogenesis}

We focused the remaining studies on a subset of genes that are involved in integrin activation and that function to promote cell adhesion and migration, given their considerable enrichment in our screen. We examined the roles of the FA protein, TLN1, and its interacting partner, FERMT2, as well as ECM ligands, VTN and LAMB4, which are preferentially recognized by integrin $\beta 1$ and other integrin combinations (Schvartz et al., 1999; Belkin and Stepp, 2000). We also included ITGB1 and Rac1 in our analysis, given their central role in the network (Figure $2 \mathrm{C}$ ). To extend the results of our screen and rule out potential off-target effects, we knocked down each of these candidates using shRNAs distinct from those used in our screen. First, we silenced TLN1, LAMB4, FERMT2, VTN, ITGB1, and Rac1 in Hs578T cells and confirmed the efficiency of knockdown by quantitative PCR (qPCR; Figure 3, A and B). As expected from our screen, ablation with shRNAs significantly increased the number of ciliated cells, and TLN1, FERMT2, and VTN depletion had the most profound effects, resulting in populations in which $\sim 30 \%$ of cells assembled cilia. By comparison, serum starvation, a potent inducer of ciliation in normal cells, typically resulted in a population with considerably less cilia ( 15-20\%; Figure 3C). We also effectively suppressed expression of each gene in RPE1 cells, as this is the most well-studied and robust in vitro model for studying mechanisms of ciliogenesis. The absence of each of these proteins substantially increased the number of ciliated cells, as observed for Hs578T cells (Figure 3, D and E). Further, we showed that expression of an RNAi-resistant FERMT2 cDNA rescued enhanced ciliation after FERMT2 depletion (see Figure 7, A and B later in the paper), confirming that the enhanced ciliation phenotype was not a result of an off-target or nonspecific effect. Interestingly, in both cell lines, knockdown of ITGB1 or Rac1 produced similar results, confirming that the integrin and Rac1 signaling pathways are linked to ciliogenesis.

Since ciliation is normally associated with cell cycle exit, we examined the proliferative status of cells depleted of each protein. With the exception of TLN1, depletion of these proteins in Hs578T did not significantly reduce the number of Ki67-positive cells, unlike serum withdrawal (Figure 3F). In striking contrast, we found that the number of proliferative, Ki67-positive RPE1 cells markedly decreased after depletion of each protein, except LAMB4, concomitant with gene silencing and enhanced ciliation, suggesting that cilium assembly was linked with cell cycle exit in normal epithelial cells (Figure 3G). This suggests that within the time frame of 
A

Hs578T
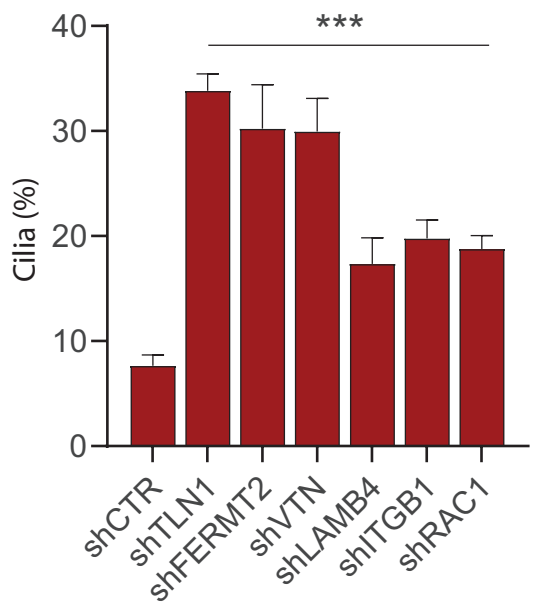

D
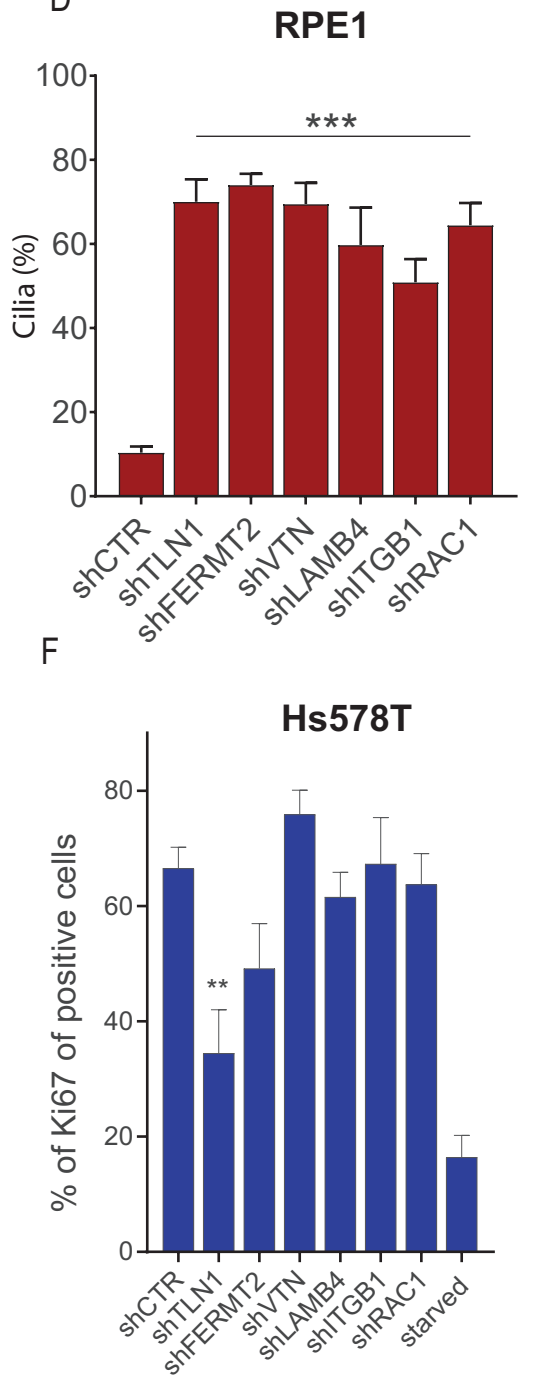

B
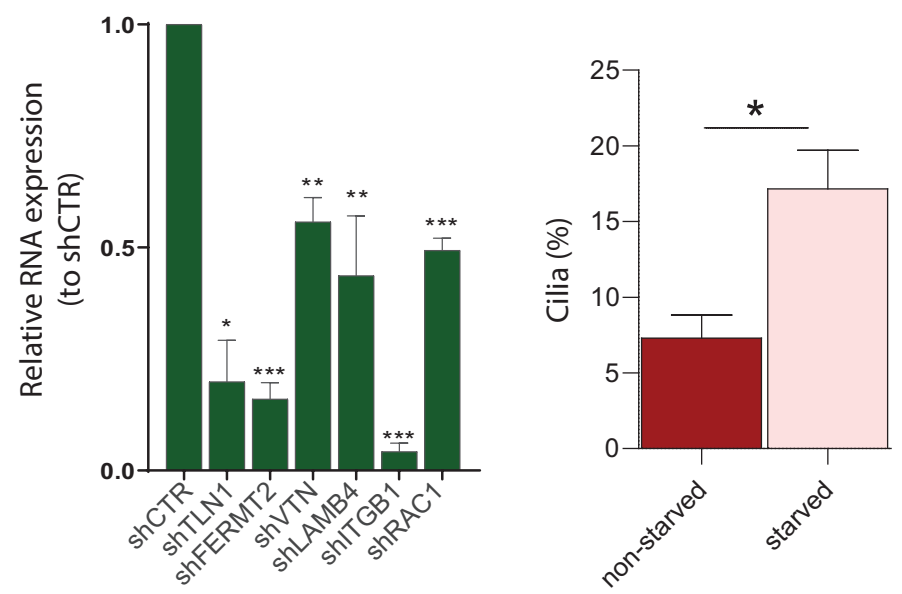

$\mathrm{E}$

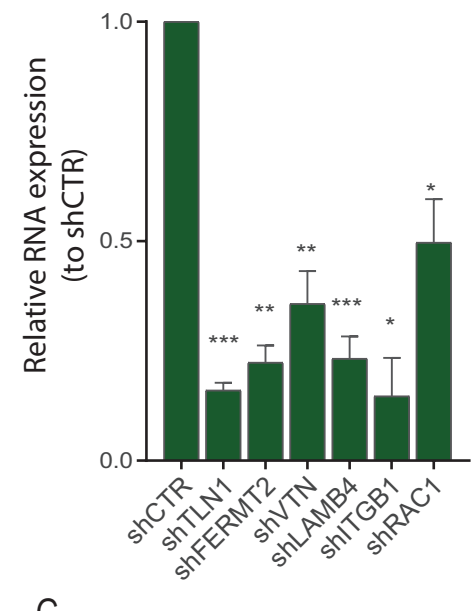

G

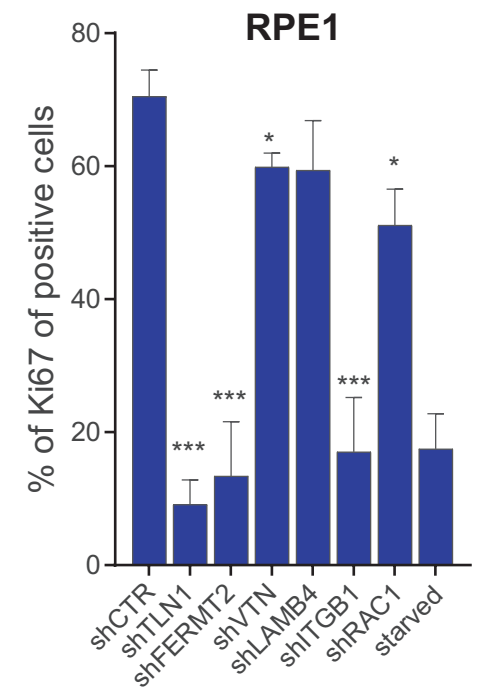

FIGURE 3: Depletion of genes within the ECM-integrin-adhesion network triggers ciliation. Hs578T or RPE1 cells treated with shRNAs against TLN1, FERMT2, VTN, (LAMB4, integrin $\beta 1$ (ITGB1), or Rac1. Depleted cells were fixed and stained to visualize cilia or Ki67, and the number of ciliated ( $A$ and $D$ ) and proliferative ( $F$ and G) Hs578T and RPE1 cells was quantified together with expression levels after depletion (B, E). (C) Ciliation of Hs578T cells was measured after serum starvation. RNA levels were measured relative to the shCTR (control) shRNA. Graphs show the mean \pm SEM of at least three independent experiments in which at least 100 cells were counted. ${ }^{\star} p<0.01,{ }^{\star \star} p<0.001$, and ${ }^{* \star \star} p<0.0001$ were calculated via contingency test. 

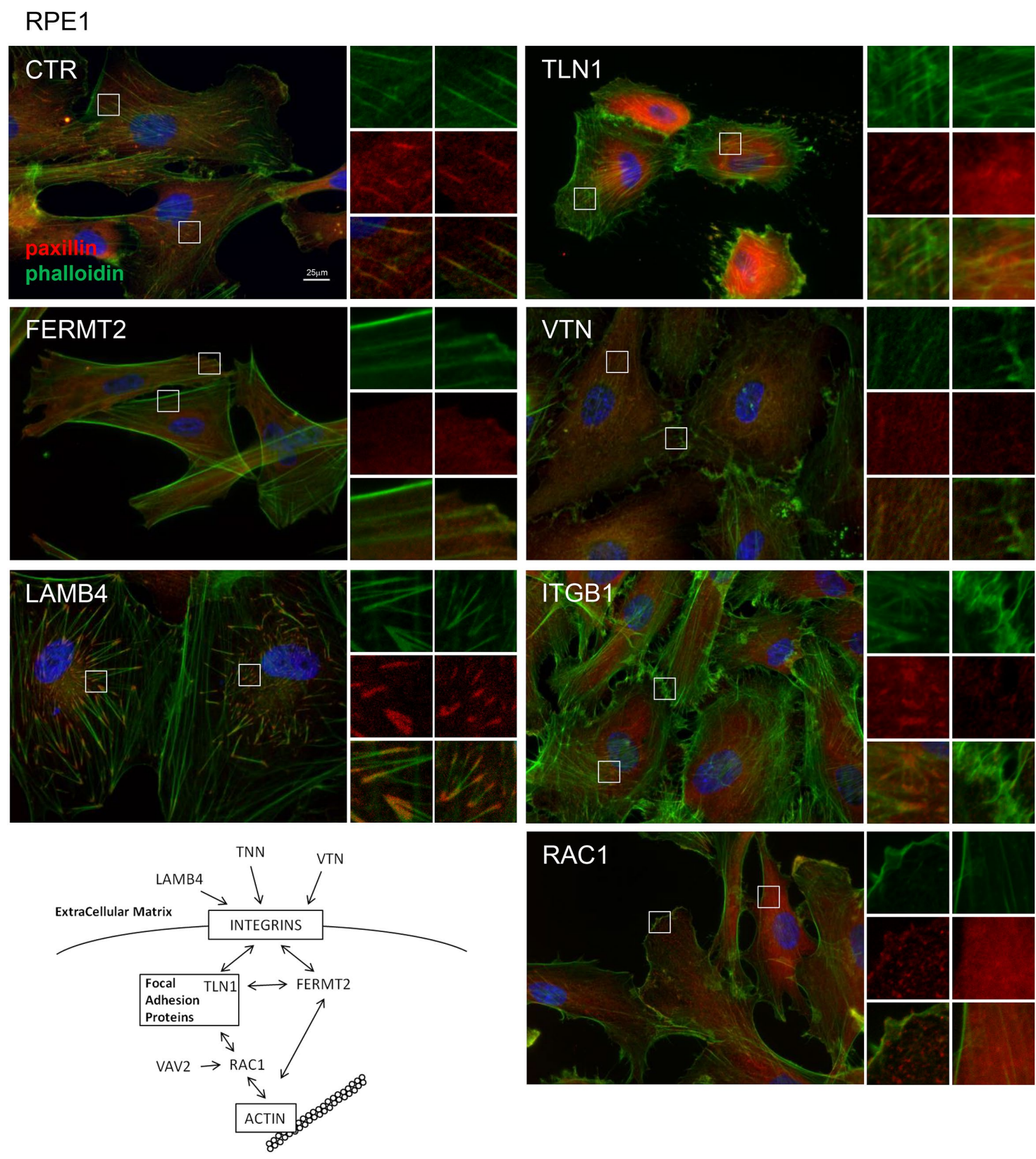

FIGURE 4: Candidate gene depletion triggers changes in adhesion and contractility. RPE1 cells treated with shRNAs against Talin1 (TLN1), FERMT2, VTN, LAMB4, integrin $\beta 1$ (ITGB1), or Rac1 were fixed and stained for actin (phalloidin, green) and paxillin (FAs, red). Insets show magnified views of actin filaments/stress fibers and FAs. Scale bar $=25 \mu \mathrm{m}$.

our experiments, suppressing genes within this network in cancer cells does not obligatorily force cell cycle exit, which could, itself, promote ciliation. This result prompts the suggestion that cell cycle exit may be disconnected from ciliation in these cells. It is also possible that genetic lesions within these cells dampen the ability of these cells to ciliate and override controls (and cell cycle exit) enforced in normal epithelial cells, although this remains to be tested.

\section{Impact of gene suppression on adhesion and contractility}

Thus far, we have shown that ablation of proteins linked to integrin and FA function can enhance ciliation. To confirm whether depletion of this subset of hits concurrently perturbed the assembly of FA complexes, RPE1 cells were stained for the FA protein, paxillin, after gene silencing (Figure 4). As expected, paxillin formed punc- tate, well-organized structures in control cells, whereas immunofluorescence of this protein appeared diffuse after TLN1, FERMT2, and Rac1 loss. Similarly, knockdown of the ECM component, VTN, elicited diffuse staining of paxillin without recruitment to FA. In contrast, paxillin puncta were segregated into thick patches at the ends of actin filaments and exhibited a random distribution after LAMB4 depletion. Surprisingly, after ITGB1 depletion, paxillin also formed thick and short patches which colocalized with the termini of internal actin stress fibers, but not at the edges of cells. Loss of integrins and FA proteins is known to perturb the actin cytoskeleton. Therefore, cells depleted of each of these proteins were also stained for actin using phalloidin. In normal cells, actin stress fibers are generally divided into three groups (Hotulainen and Lappalainen, 2006; Pellegrin and Mellor, 2007): 1) ventral stress fibers, which are anchored at both ends by FA and that are essential for 
cell contractility; 2) dorsal stress fibers, which are linked to a transverse arc at one end and to an FA at the other; and 3) transverse stress fibers, which are located under lamellipodia and are connected to FAs via dorsal stress fibers. In control RPE1 cells, phalloidin staining revealed a well-developed network of thin stress fibers, with many actin cables running longitudinally across the cell body and connected to FAs at both termini, suggesting that RPE1 cells extensively assemble ventral fibers (Figure 4). In TLN1-depleted cells, phalloidin staining was diffuse within the cytoplasm, and stress fibers appeared sparse and less organized than in controls, resulting in rounder cells suggestive of a loss of contractility. Indeed, it has already been shown that traction force generation was significantly reduced in Talin-deficient cells (Zhang et al., 2008). In FERMT2-depleted cells, internal stress fibers essentially disappeared, and phalloidin primarily marked tangential lines on the edge of cells, suggesting loss of contractility. Similar lattices were observed in RPE1 cells plated on fibronectin micropatterns (Théry et al., 2006), where stress fiber strength along edges depends on local adhesiveness: actin cables were considerably larger along nonadhesive edges than adhesive edges, confirming the absence of membrane adhesion to the substratum in FERMT2-depleted cells. Interestingly, knockdown of the integrin ligand, VTN, led to a phenotype similar to TLN1-depleted cells, wherein stress fibers were more sparse and less organized. Since actin organization is a fundamental regulator of stress fiber organization and contractility, we conclude that the dispersion of actin filaments observed in TLN1- and VTN-depleted cells perturbed cell contractility. VTNdepleted cells also exhibited an accumulation of cortical actin and a disk-like appearance, signifying an increase of cell spreading on the surface. After Rac1 depletion, cells appeared elongated and lacked detectable F-actin structures other than cortical actin, as previously described (Guo et al., 2006). By contrast, after LAMB4 or ITGB1 depletion, cells exhibited numerous, thick, and short actin fibers between FA, suggesting an accumulation of ventral stress fibers and an increase in contractility. The mechanical force that is transmitted to FAs by stress fibers can alter the FA complex, and the augmentation of mechanical forces in LAMB4- and ITGB1-depleted cells may explain the increase in FA size described earlier. Thick stress fibers are usually found in nonmotile cells and are relatively stable. Indeed, cells depleted of LAMB4 and ITGB1 also exhibited enlarged nuclei and cytoplasm with a large and flattened disk-like appearance, signifying enhanced cell spreading.

We sought to further investigate the link between altered stress fibers and enhanced ciliogenesis. Increased RhoA was shown to induce stress fiber formation and prevent primary cilium assembly (Dawe et al., 2009), and therefore we examined the impact of specifically blocking ROCK with Y27632 to inhibit cell contractility. As expected, treatment of control RPE1 cells with this drug induced actomyosin relaxation, as evidenced by changes in cell morphology and enhanced ciliogenesis (Figure 5, A and B). In contrast, cells depleted of LAMB4, Rac1, or ITGB1 showed no further or substantial increases in ciliation (Figure 5B). Because treatment with this drug did not additively increase the frequency of ciliation, we conclude that these candidate genes most likely suppress cilium assembly by inducing changes in cell contractility. Alternatively, it is possible that depletion of proteins such as LAMB4 increased ciliation to maximal levels in a contraction-independent manner.

\section{Restoration of ECM ligand rescues ciliogenesis}

Interestingly, our results suggest that ciliation of normal and breast cancer cells is regulated in part by the integrin ligands produced by these cells. Depletion of either VTN or LAMB4 led to marked enhancements in ciliation of normal and breast cancer cells (Figure $3, A$ and D). To further test the conclusion that extracellular levels of these proteins were critical for such regulation, we supplied VTN to the ECM of Hs578T cells depleted of this protein by plating cells on coverslips with different concentrations of ligand. We found that the addition of purified VTN reversed the impact of depletion with the shRNA and significantly reduced the frequency of ciliation in these cells, whereas the addition of a control protein (bovine serum albumin; BSA) had no effect (Figure 5C). These findings strongly support our conclusion that the presence of extracellular matrix proteins can dramatically alter the ability of cells to ciliate.

\section{Targeting ciliogenesis inhibitors impairs cell migration}

We next evaluated the impact of silencing candidate genes on cell migration by examining whether assembly of cilia plays a role in this process. We therefore ablated a subset of proteins (VTN, FERMT2, LAMB4, Rac1, and TLN1) in Hs578T cells using shRNAs and performed wound-healing assays. We found that suppression of TLN1, FERMT2, and LAMB4 markedly reduced migration of these cancer cells over a $\sim 22-h$ window without substantially decreasing their proliferative capacity during this period (Figure 6A). These results suggest that enhanced ciliation of breast cancer cells coincided with reduced cell migration.

To generalize our findings, we also ablated TLN1, LAMB4, FERMT2, VTN, and Rac1 in RPE1 cells using individual siRNAs distinct from those used in the screen, and cells were allowed to reach confluence for a short duration before wounding. In each case, we observed a significant increase in the percentage of ciliated cells as compared with the siRNA control, although the extent of ciliation was somewhat lower than that obtained using shRNAs (Supplemental Figure 3B). Importantly, we found that cell migration was significantly impaired after ablation of all candidates in RPE1 cells (Figure 6C and Supplemental Figure 3A). To test whether the impact on cell migration resulted from enhanced ciliation, we simultaneously depleted CEP83, a centriolar distal appendage protein essential for ciliogenesis (Tanos et al., 2013) and confirmed silencing with qPCR (Supplemental Figure 3B). Removal of CEP83 abolished ciliation, as expected (Supplemental Figure 3B). Importantly, migration was rescued to levels approximating the control after simultaneous ablation of CEP83 and TLN1, VTN, or Rac1, indicating that cilium assembly can restrain cell migration (Figure 6C).

We conclude that assembly of cilia restricts cell migration in normal epithelial and cancer cells. These results stand in contrast with fibroblasts, wherein migrating cells assembled cilia oriented in the direction of cell movement (Christensen et al., 2013), suggesting that the role of cilia in migration could be cell type dependent.

\section{YAP/TAZ pathway activation can rescue aberrant ciliation triggered by depletion of a FA-integrin pathway component} Last, we sought a mechanistic explanation for the induction of ciliation on disruption of the FA complex. The Hippo pathway effectors, YAP/TAZ, are transcriptional coactivators shown to function as mechanosensitive switches by responding to changes in the ECM and FAs and altering expression of genes encoding FA proteins and negative regulators of cilium assembly (Kim et al., 2015a; Nardone et al., 2017). Furthermore, actin destabilization was shown to induce ciliation by disabling YAP/TAZ activity through cytoplasmic sequestration, whereas hyperactivation of YAP can suppress serum starvation-induced ciliation (Kim et al., 2015a). To test the involvement of the YAP/TAZ pathway in responding to loss of our candidate genes, we assessed the impact of silencing FERMT2, since FERMT2 and 


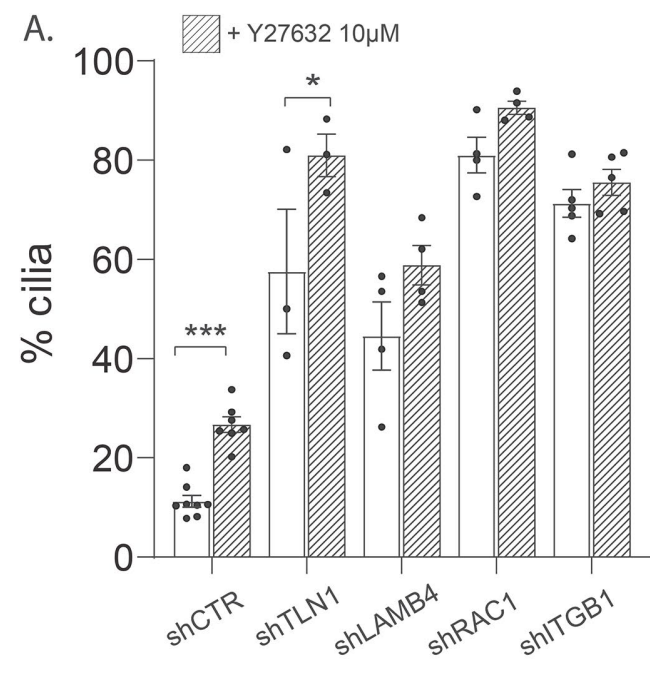

B.
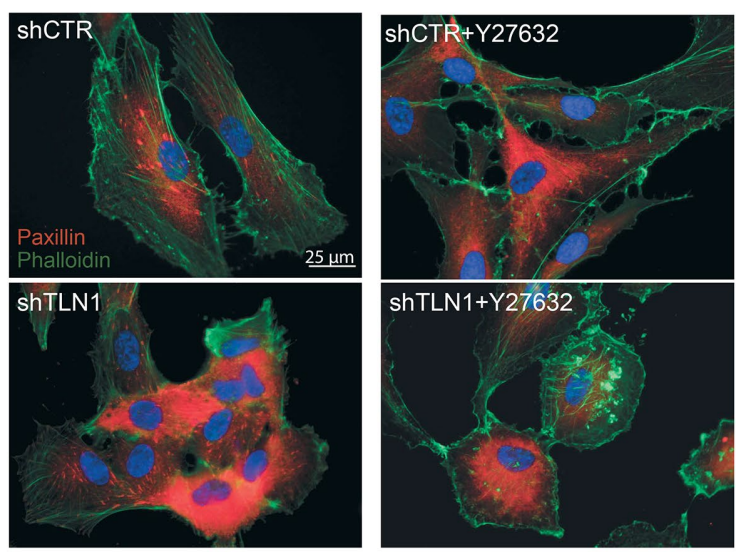

C.
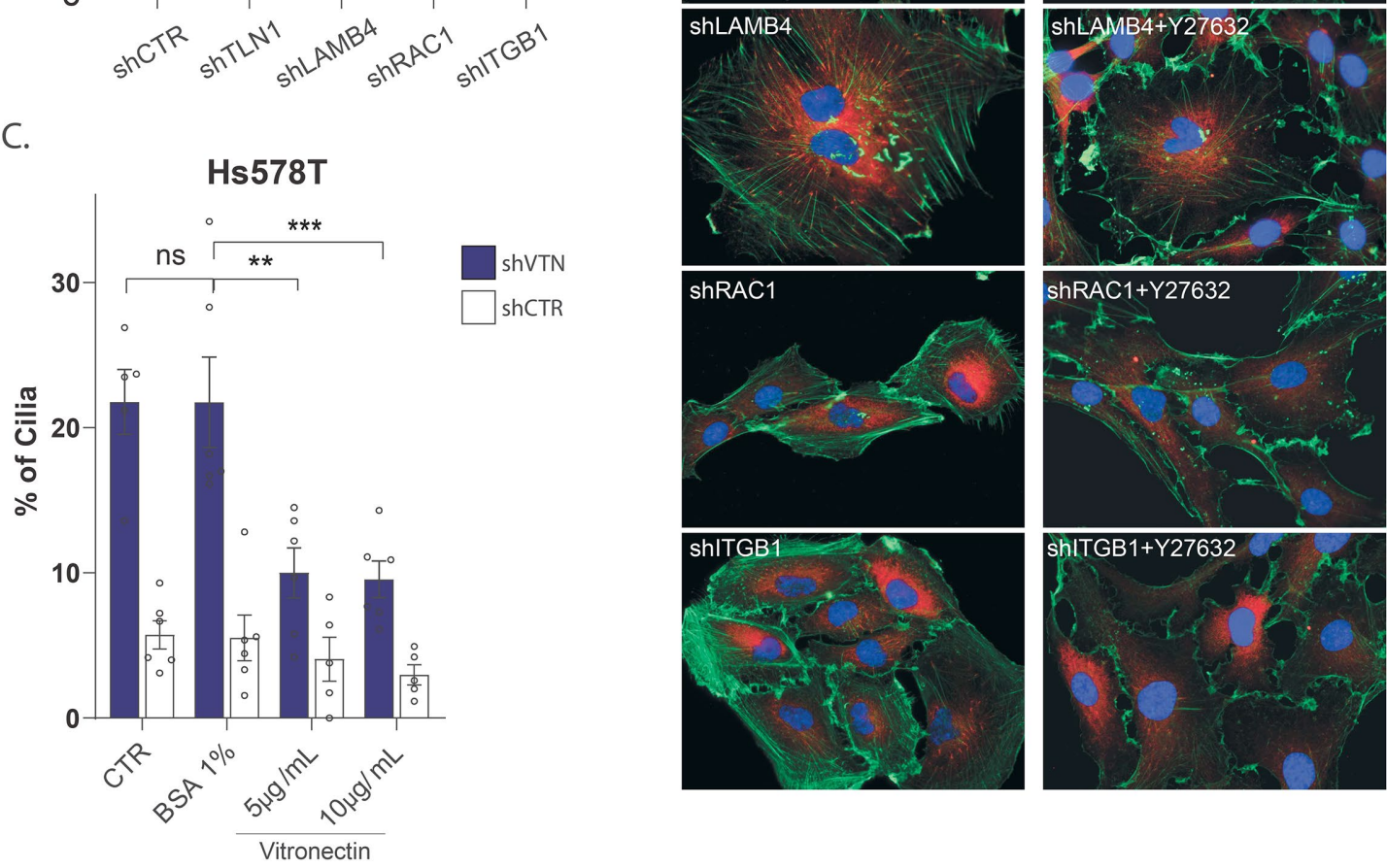

FIGURE 5: Impact of gene silencing on adhesion and contractility. (A) RPE1 cells treated with shRNA control (shCTR) or shRNA against Talin1 (TLN1), FERMT2, VTN, integrin $\beta 1$ (ITGB1), or Rac1 were incubated with the Rho kinase inhibitor, Y27632 (hatched bars). Cilia were stained with antibodies against Arl13b and quantified. Graph shows the mean \pm SEM of at least three independent experiments in which at least 100 cells were counted. (B) Actin and FA were stained with phalloidin (green) and paxillin (red), respectively. (C) Hs578T cells depleted for VTN were plated on coverslips supplied with 5 or $10 \mu \mathrm{g} / \mathrm{ml}$ purified vitronectin or BSA as control. Cilia were stained and quantified as indicated. In each case, the graph shows the mean \pm SEM of at least three independent experiments in which at least 100 cells were counted.

another protein, TLN1, bind to the cytoplasmic tail of $\beta$-integrins to enable their activation via ECM ligands (Kim et al., 2011). We expressed a hyperactivated mutant YAP protein, in which all inactivating phosphorylation sites were eliminated (GFP-YAP5SA; Zhao et al., 2007; Kim et al., 2015a), or GFP as a control, in RPE1 cells, then suppressed expression of FERMT2. As shown in Figure 7, A and $B, F E R M T 2$ knockdown in control cells induced robust levels of ciliation, comparable to what was observed in the parental RPE1 cell line (Figure 3D). Interestingly, we observed substantially reduced levels of ciliation in cells expressing constitutively active GFP-YAP as compared with the control. Thus, activated YAP can rescue the enhanced ciliation induced through FERMT2 knockdown. FERMT2 loss can compromise integrin activation, which is required to promote and stabilize the assembly of the actin cytoskeleton. Since FERMT2 depletion negatively impacts actin network assembly (Figure 4), our screen suggests that such destabilization promotes ciliogenesis, and YAP/TAZ components can override this response by enhancing expression of genes mediating integrin-dependent FA assembly and possibly other genes that counteract ciliogenesis (Kim et al., 2015a).

Our studies have identified a cohort of genes that suppress ciliogenesis through heretofore unanticipated processes. We suggest that our screen may have uncovered an expansive set of genes that could ultimately be implicated in genetic diseases with ciliopathic features. As exome sequencing of rare mutations proceeds, it will be interesting to query the linkage of disease mutations using our list of candidate ciliogenesis suppressors. Importantly, our results also suggest that FAs impact ciliogenesis through an integrin-associated pathway. Such modulation could occur through reorganization of the actin network, and our work significantly extends the repertoire of proteins that may play a role in the decision to assemble cilia. For example, we have definitively shown that altering cell 
A

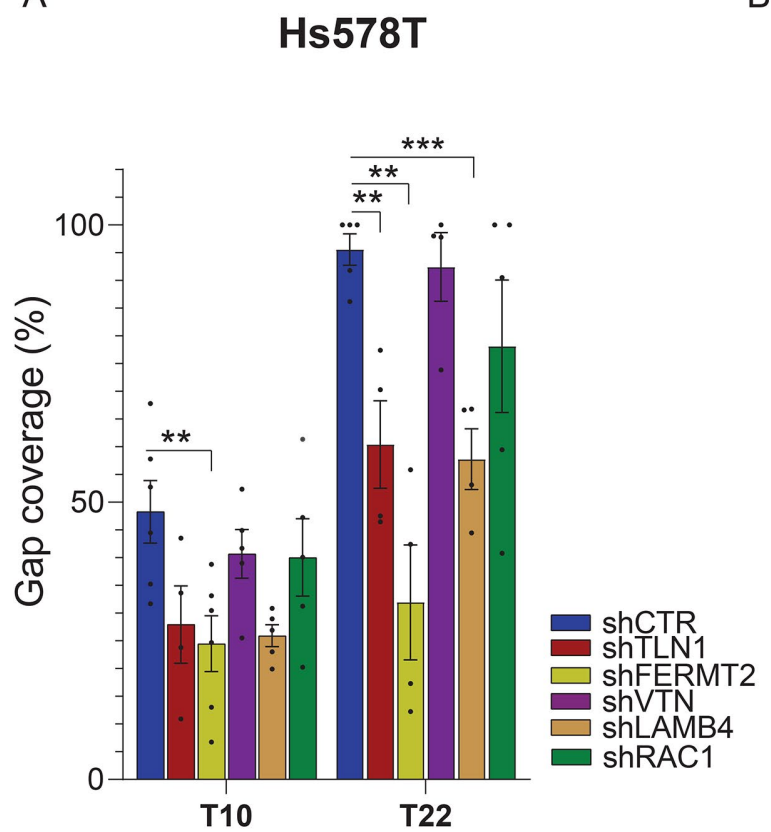

Time after scratch (hours)
B
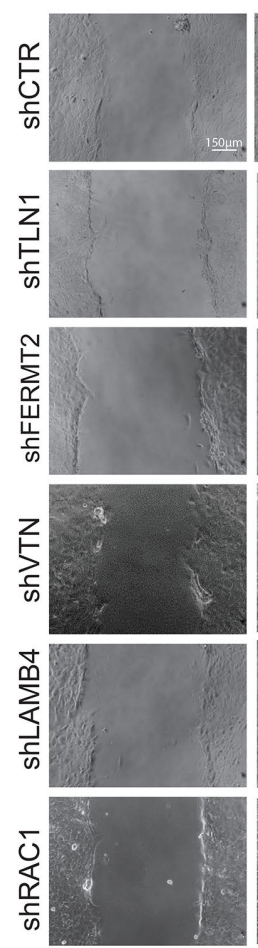

$\mathrm{T} 10$
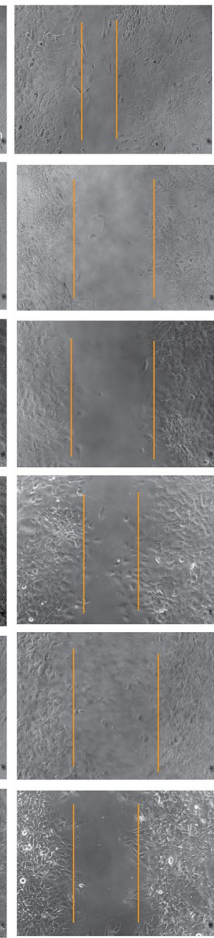

T22
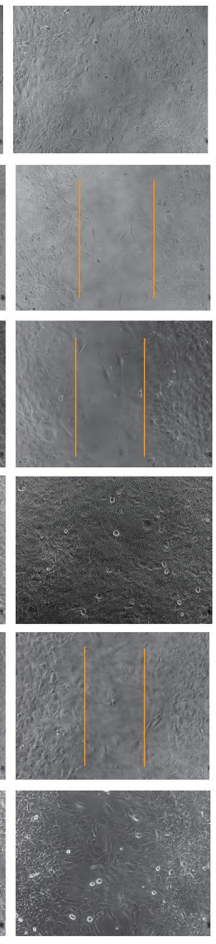

C

\section{RPE1}
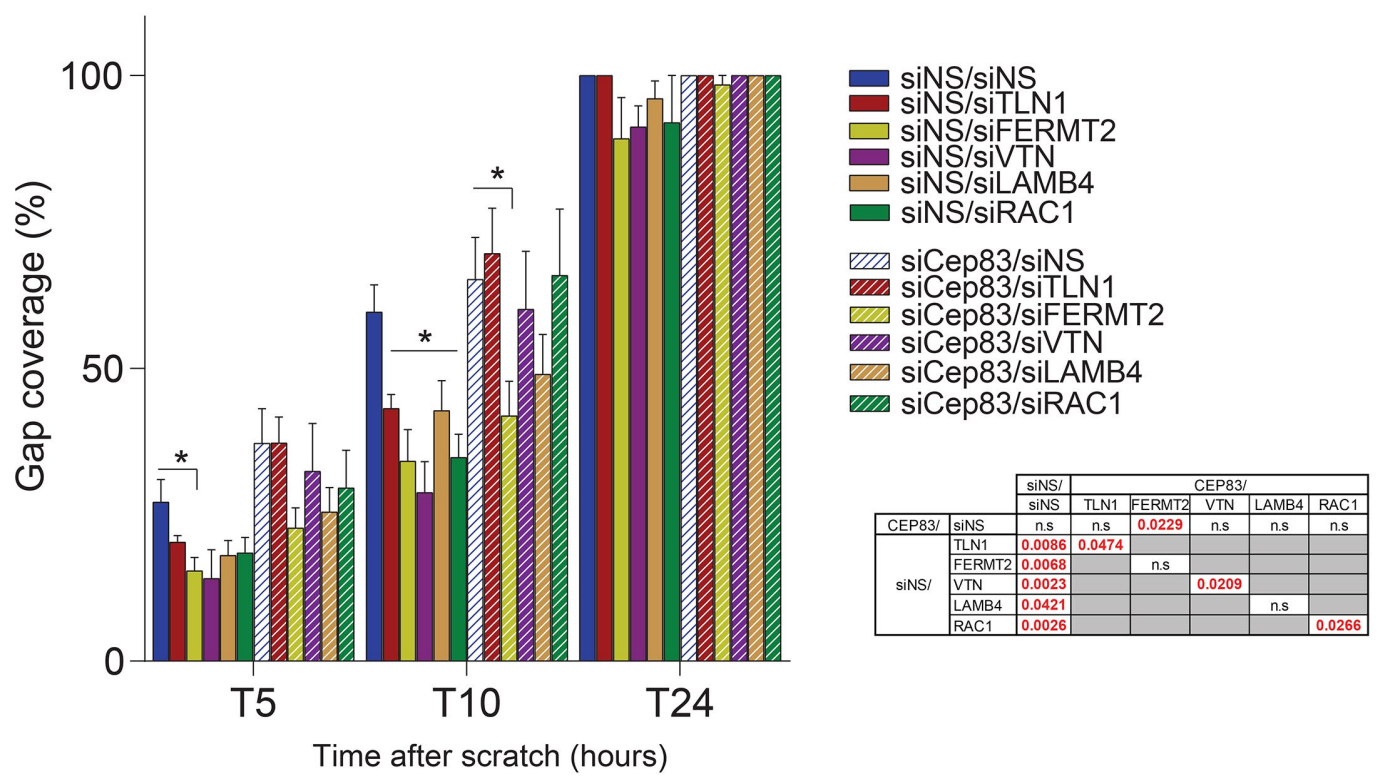

FIGURE 6: Enhanced ciliation restrains cell migration. (A) Wound healing assay was performed on $\mathrm{Hs} 578 \mathrm{~T}$ cells treated with shRNAs against Talin1 (TLN1), FERMT2, VTN, LAMB4 or Rac1 in. Cells were imaged $10 \mathrm{~h}$ and $22 \mathrm{~h}$ after the scratch, the gap size was measured and its rate of closure was calculated over time. (B) Wound healing assays were performed in RPE1 cells treated with siRNAs against CEP83 and Talin1 (TLN1), FERMT2, VTN, LAMB4 or Rac1 in serum replete conditions. The gap size was measured 5,10 , and $24 \mathrm{~h}$ after the scratch, and its rate of closure was calculated over time. Graph shows the mean \pm SEM of at least three independent experiments in which at least 100 cells were counted.

attachments to the ECM through FAs modifies the dynamics of primary cilium assembly, which could further serve to modulate cellular responsiveness and migration during malignant transformation and metastasis. Since ciliation and cell-ECM interactions are perturbed in the epithelial cells of basal breast cancers, restoring cytoskeletal organization and proper interactions with the ECM might rescue ciliary intra- and extracellular signaling pathways and cell proliferation, preventing the metastatic progression of primary epithelial 
A.
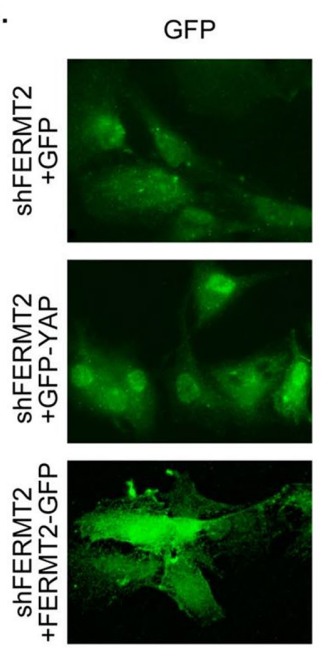

GT335
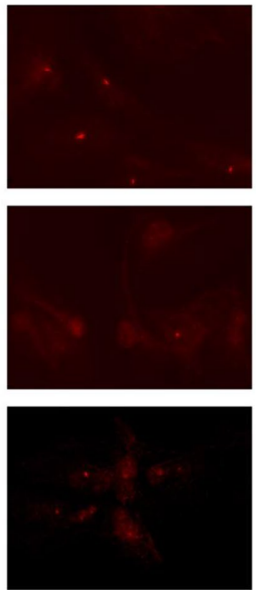
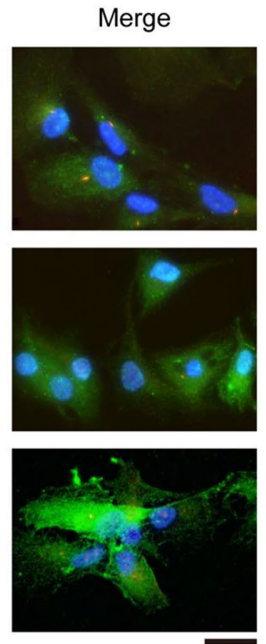

B.

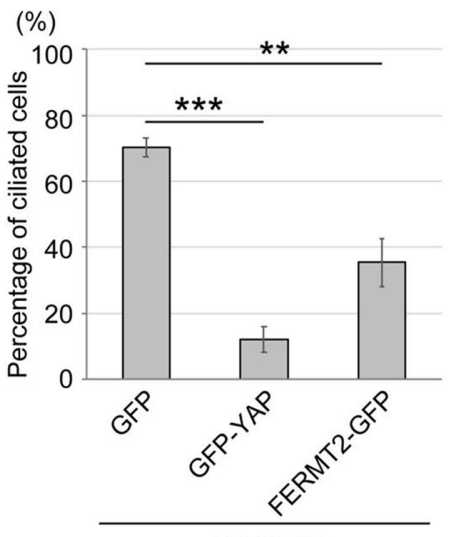

shFERMT2

C.

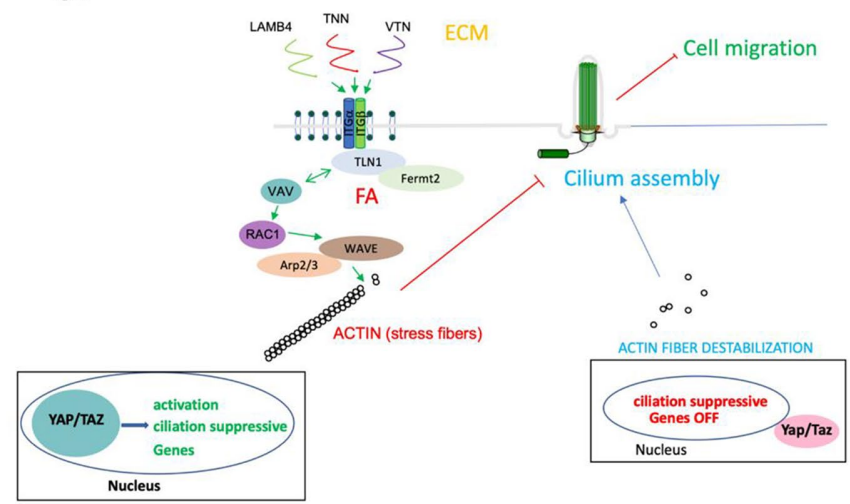

FIGURE 7: Constitutive activation of YAP/TAZ pathway rescues depletion of Fermt2. (A) FERMT2 was depleted using shRNA in RPE-1 cells stably expressing GFP, GFP-YAP or FERMT2-GFP. Cells were immuno-stained with anti-GT335 to detect cilia and with anti-GFP antibodies (A). (B) Percentages of ciliated cells shown in A were quantified (three independent experiments). Error bars represent SD. The $p$ values were calculated by Scheffe's Test after ANOVA $\left({ }^{\star *} p<\right.$ $0.001,{ }^{* \star} p<0.0001$ ). (C) Model indicating the role of ECM and FA components and YAP/TAZ pathway in restraining cilium assembly. See text for details.

cancers in aggressive solid tumors such as TNBC. We also showed that modulating the concentration of certain ligands in the ECM impacts the ability of cells to ciliate and migrate, and this finding confirms the results of our screen and, importantly, suggests that the extent and efficacy of ciliation could be impacted in a highly context-specific manner by local microenvironments within the ECM and adhesive interactions. Interestingly, recent studies have shown that removal of cilia was associated with mitral valve prolapse and reorganization of components within the ECM (Toomer et al., 2019), potentially indicating a reciprocal relationship between ciliation and the integrity of the ECM. Our work provides a valuable resource for pursuing these connections.

While we have shown that ablation of several genes identified in our screen could trigger both cell cycle exit and cilium assembly in normal epithelial cells, wholesale cell cycle exit was not induced by depletion of these proteins in basal cancer cells over the time course used in our study. Because cell cycle exit is normally intricately linked to cilium assembly, we anticipate that the timing and duration of protein depletion could impact the outcome, and more prolonged depletion may be necessary to enforce growth arrest. It is also possible that breast cancer cells are refractory to growth arrest owing to additional genetic alterations. Since it would be advantageous to enforce growth arrest in a cancer cell that has lost the primary cilium, inhibition of this ciliary suppression program with small molecules may therefore provide unique opportunities for attenuating cell proliferation and arresting tumor development. We note that a significant number of hits in our screen have enzymatic properties, suggesting that tumor cells could be reciliated by novel (or even preexisting) small molecule inhibitors. Furthermore, we surmise that, unlike toxic chemotherapies used currently, reciliation of cancer cells may not pose detrimental effects organismically, since cells that otherwise lack the machinery to ciliate (such as hematopoietic or muscle cells) are not likely to be adversely affected by induced ciliation. On the other hand, the ability to reciliate basal breast cancer cells could constitute an Achilles' heel for slowing growth of this type of tumor.

\section{MATERIALS AND METHODS \\ Cell culture}

Normal RPE1 cells and basal breast cancer cells (Hs578T, MDAMB-157, and MDA-MB-468) obtained from ATCC were cultured in DMEM supplemented with $10 \%$ fetal bovine serum (FBS) under standard conditions $\left(37^{\circ} \mathrm{C}, 5 \% \mathrm{CO}_{2}\right)$. Normal breast MCF10A cells were cultured in DMEM medium supplemented with 10\% FBS, epidermal growth factor $(20 \mathrm{ng} / \mathrm{ml})$, cholera toxin $(100 \mathrm{ng} / \mathrm{ml})$, insulin 
$(0.01 \mathrm{mg} / \mathrm{ml})$, and hydrocortisone $(500 \mathrm{ng} / \mathrm{ml})$ under standard conditions $\left(37^{\circ} \mathrm{C}, 5 \% \mathrm{CO}_{2}\right)$.

\section{Whole-genome and custom siRNA library screens}

The human Silencer Select (Thermo Fisher Scientific) arrayed siRNA library containing pooled siRNAs targeting $\sim 22,000$ human genes was screened in triplicate. Assay plates (384-well plate with optical bottom; Corning 3712) were spotted with $1.5 \mu$ of $0.3 \mu \mathrm{M}$ siRNA using the Agilent Bravo automated liquid handler with a 384-tip head. Reverse transfection was performed using Lipofectamine RNAiMAX, and the final siRNA concentration was $33 \mathrm{nM}$. Hs578T cells were suspended in DMEM supplemented with 10\% FBS and seeded onto assay plates using the Matrix Well Mate (2000 cells in $40 \mu \mathrm{l}$ medium for each well). Culture medium was replaced with DMEM $24 \mathrm{~h}$ after transfection using the Biotek EL406, and cells were incubated for an additional $72 \mathrm{~h}$ before fixation in $4 \%$ formaldehyde, blocking in PBS/3\% BSA/0.1\% Triton, and subsequent staining with Arl13b (1:10,000; 75-287, NeuroMab) in blocking buffer, anti-mouse Alexa 647 (1:20,000; Invitrogen), and DAPI. For the custom siRNA library screen, an arrayed library containing the same pooled siRNAs targeting 349 human genes identified in the whole-genome screen was screened and processed as described above.

\section{Imaging and image analysis}

Concurrent image acquisition and analysis of the siRNA screen was performed on the ArrayScan VTI (Thermo Fisher Scientific) using the Spot Detector (v4) Bioapplication within the HCS Studio Cell Analysis suite. Briefly, 6 fields/well with 2 channels/field (DAPI, AF647) were imaged at $20 \times$ magnification and $2 \times 2$ binning $(1104 \times 1104$ resolution). Nuclei as primary objects were identified using an isodata thresholding algorithm and selected as valid objects by applying a minimum area criterion to reject fragmented nuclei. ARL13b staining (cilia) was identified within a circular region of 35 pixels around the nuclear mask, applying 3-sigma thresholding algorithm in the AF647 channel.

\section{Analysis of primary screen}

The statistical significance of effects on cilia and cell number after siRNA knockdown was tested by calculating robust $z$ scores from all observed cilia/cell ratios within the plate. We used the robust (Rob) $z$ score, which substitutes the outlier-insensitive median and MAD for mean and SD in the $z$ score calculation: Rob Z score $=$ (measurement - Med)/MAD. The Rob Z score was calculated for this RNAi screen because it is less sensitive to outliers (Birmingham et al., 2009). To exclude siRNAs affecting cell survival and cell cycle arrest, cell numbers after depletion of KIF11 (involved in spindle dynamics during mitosis) and NEK2 (required for mitotic progression) were used to set a cell number cutoff which corresponded to the lowest number of cells in NEK2-depleted control cells (zcell cutoff $=180$ ).

The range of robust $z$ scores over the course of the screen was consistent, but to ensure normalization of data and exclusion of batch-specific effects, data were analyzed within processed batches. The zcilia cutoff was set at a robust $z$ score $=3$. Some batches showed a lower ciliation efficiency, and the cutoff was set according to the median and the MAD of robust $z$ scores for the positive controls (Med zcilia ctl + 1.45*MAD zcilia ctl = zcilia cutoff) (Zhang, 2011). If a test result was greater in value than the median robust $z$ score for the positive controls (zcilia > zcilia cutoff) for at least two replicates, then this siRNA has a greater effect on ciliogenesis than the median of the positive controls, and the result was considered as a significant hit.
Finally, to control the rate at which siRNAs that have true large or moderate effects fail to be identified as positives and the rate at which siRNAs that should be considered negative are identified as positives, hits with a SSMD > 1.645 were selected (Zhang, 2011).

\section{Confirmatory screen}

The statistical significance of effects on cilia and cell number after siRNA knockdown was tested by calculating robust $z$ scores from the ratio of cilia/cell number in negative control wells on the plate. To exclude siRNAs affecting cell survival, an initial cutoff was set according to the number of RPE1 (zcell cutoff $=400$ ) and MDA-MB-468 (zcell cutoff $=800$ ) cells. The zcilia cutoff was set at Rob Z score $=3$ for all cell lines, except a Rob $Z$ score $=1.5$ was used for MDAMB-157 cells.

\section{RNA interference}

To confirm the results of primary and secondary screens, we selected shRNAs from the MISSION pLKO.1-puro lentivirus library (Sigma-Aldrich). Plasmids were transfected in HEK293T cells with delta 8.2 and VSV-G vectors and polyethylenimine (PEI) for 3 to $4 \mathrm{~d}$.

Virus was filtered, and RPE1 cells were infected with fresh media (ratio 1:1) and polybrene for 8-12 h. Cells were selected with puromycin $(6.67 \mu \mathrm{g} / \mathrm{ml}$ for RPE1; $1 \mu \mathrm{g} / \mathrm{ml}$ for Hs578T) for 1 day. Then, cells were trypsinized, replated, and analyzed after $2 \mathrm{~d}$.

Cells were reverse-transfected with $30 \mathrm{nM}$ siRNA and Lipofectamine (RNAiMax; Invitrogen) for $8 \mathrm{~h}$. Analysis of cilia was performed $96 \mathrm{~h}$ after transfection. Sequences used are shown in Supplemental Table S1.

\section{Immunofluorescence}

Cells were fixed with $4 \%$ formaldehyde for $10 \mathrm{~min}$ at room temperature. After blocking in PBS/3\% BSA/0.2\% Triton-X, cells were immunostained either with antibodies against ARL13b and Ki67 or with paxillin and phalloidin for $2 \mathrm{~h}$. Secondary antibodies were incubated for $2 \mathrm{~h}$, and cilia and Ki67-positive nuclei were quantified in 100 cells for each replicate.

Statistical contingency and $\chi^{2}$ testing were performed with data from at least three replicates.

\section{Flow cytometry}

Flow cytometric analyses with anti-Ki67-FITC antibody (Miltenyi Biotec) was performed following manufacturer's instructions. Briefly, cells were fixed in cold ethanol (70\%) for at least $2 \mathrm{~h}$. After washing, Ki67 antibody was diluted 1/50 in incubation buffer (PBS/3\% BSA $+0.3 \%$ Triton-X) and incubated for $20 \mathrm{~min}$ at room temperature. Ten thousand cells were counted using the LSRII UV cell analyzer (BD Bioscience) and FlowJo software.

Experiments were performed a minimum of three times.

\section{Migration assays}

Eighty thousand RPE1 cells were seeded in a 24-well plate. Cells were reverse-transfected at the time of seeding with siRNAs targeting candidate genes and control or CEP83 siRNAs. After $72 \mathrm{~h}$, cells were wounded using a 10- $\mu \mathrm{l}$ tip, and gap closure was monitored at 5, 10, and $24 \mathrm{~h}$. For Hs578T, 100,000 cells infected with lentivirus harboring each shRNA and selected with puromycin were seeded in a 24well plate. After $48 \mathrm{~h}$, cells were wounded as above, and gap closure was monitored at 10 and $22 \mathrm{~h}$. The area encompassing the gap was measured using ImageJ software and presented as a percentage of gap closure compared with Time 0 . Experiments were performed minimally three times with at least two technical replicates each. 


\section{Expression of an siRNA-resistant cDNA or activated YAP in Fermt2-depleted cells}

To determine whether we could specifically rescue enhanced ciliation as a result of depletion of FERMT2, we selected RPE1 cell lines stably expressing GFP or an siRNA-resistant FERMT2-GFP. We also expressed a hyperactivated YAP mutant (GFP-YAP5SA) in which all inactivating phosphorylation sites were eliminated (Zhao et al., 2007; Kim et al., 2015a). We suppressed expression of Fermt2 in each of these cell lines by infection with a lentivirus expressing a FERMT2 shRNA that targets a region within the 3' UTR of the FERMT2 gene (Supplemental Table S3). Virally transduced cells were selected and then fixed. Ciliation was then scored by immunostaining with the anti-polyglutamylation antibody, GT355. A total of 100 cells were scored in each case, and the experiment was performed three times.

\section{Gene expression analysis}

Total RNA was isolated using TRlzol Reagent (Fisher). RNA was reverse-transcribed using Verso cDNA synthesis kit (Thermo Scientific). Quantitative reverse-transcription PCR (RT-qPCR) was performed using the CFX96 Touch Real-Time PCR Detection System (Bio-Rad). Relative expression levels were calculated with the $\Delta \Delta \mathrm{Ct}$ method using GAPDH as an internal control. Primer sequences are shown in Supplemental Table S1.

\section{ACKNOWLEDGMENTS}

We thank J. Reiter and L. Wang and other members of the Dynlacht laboratory for advice and technical assistance. We thank D-S. Lim and J. Kim (KAIST, South Korea) for YAP-5SA. This work was supported by grants from the National Institutes of Health (1R21CA207659 and 9R01GM120776) to B.D.D. and I.S. (1R21CA220518) and from the Breast Cancer Research Foundation to F.E. We acknowledge support from the High Throughput Biology Core at the NYU School of Medicine, wherein the whole-genome screen was performed.

\section{REFERENCES}

Belkin AM, Stepp MA (2000). Integrins as receptors for laminins. Microsc Res Tech 51, 280-301.

Besson A, Wilson TL, Yong VW (2002). The anchoring protein RACK1 links protein kinase Cepsilon to integrin beta chains. Requirements for adhesion and motility. J Biol Chem 277, 22073-22084.

Bhadriraju K, Yang M, Alom Ruiz S, Pirone D, Tan J, Chen CS (2007). Activation of ROCK by RhoA is regulated by cell adhesion, shape, and cytoskeletal tension. Exp Cell Res 313, 17673200.

Bijian K, Lougheed C, Su J, Xu B, Yu H, Wu JH, Riccio K, Alaoui-Jamali MA (2013). Targeting focal adhesion turnover in invasive breast cancer cells by the purine derivative reversine. Br J Cancer 109, 2810.

Birmingham A, Selfors LM, Forster T, Wrobel D, Kennedy CJ, Shanks E, Santoyo-Lopez J, Dunican DJ, Long A, Kelleher D, et al. (2009). Statistical methods for analysis of high-throughput RNA interference screens. Nat Methods 6, 569-575.

Burkhalter MD, Sridhar A, Sampaio P, Jacinto R, Burczyk MS, Donow C, Angenendt M, Hempel M, Walther P, Pennekamp P, et al. (2019). Imbalanced mitochondrial function provokes heterotaxy via aberrant ciliogenesis. J Clin Invest 129.

Christensen ST, Veland IR, Schwab A, Cammer M, Satir P (2013). Analysis of primary cilia in directional cell migration in fibroblasts. In: Methods in Enzymology, San Diego: Academic Press, 45-58.

Dawe HR, Adams M, Wheway G, Szymanska K, Logan CV, Noegel AA, Gull $K$, Johnson CA (2009). Nesprin-2 interacts with meckelin and mediates ciliogenesis via remodelling of the actin cytoskeleton. J Cell Sci 122, 2716-2726

Delyon J, Servy A, Laugier F, André J, Ortonne N, Battistella M, Mourah S, Bensussan A, Lebbé C, Dumaz N (2017). PDE4D promotes FAK-mediated cell invasion in BRAF-mutated melanoma. Oncogene 36, 3252-3262.
Dhanasekaran N, Dermott JM (1996). Signaling by the G12 class of G proteins. Cell Signal 8, 8842523.

García-Zaragoza E, Pérez-Tavarez R, Ballester A, Lafarga V, Jiménez-Reinoso A, Ramírez A, Murillas R, Gallego MI (2012). Intraepithelial paracrine Hedgehog signaling induces the expansion of ciliated cells that express diverse progenitor cell markers in the basal epithelium of the mouse mammary gland. Dev Biol 372, 28-44.

Goetz SC, Anderson KV (2010). The primary cilium: a signalling centre during vertebrate development. Nat Rev Genet 11, 331-344.

Gomez-Rodriguez J, Readinger JA, Viorritto IC, Mueller KL, Houghtling RA, Schwartzberg PL (2007). Tec kinases, actin, and cell adhesion. Immunol Rev 218, 45-64.

Goodman L, Zallocchi M (2017). Integrin $\alpha 8$ and Pcdh15 act as a complex to regulate cilia biogenesis in sensory cells. J Cell Sci 130, 36983712.

Gopalakrishnan K, Kumarasamy S, Abdul-Majeed S, Kalinoski AL, Morgan EE, Gohara AF, Nauli SM, Filipiak WE, Saunders TL, Joe B (2012). Targeted disruption of Adamts16 gene in a rat genetic model of hypertension. Proc Natl Acad Sci USA 109, 20555-20559.

Guo F, Debidda M, Yang L, Williams DA, Zheng Y (2006). Genetic deletion of Rac1 GTPase reveals its critical role in actin stress fiber formation and focal adhesion complex assembly. J Biol Chem 281, 18652-18659.

Gupta GD, Coyaud É, Gonçalves J, Mojarad BA, Liu Y, Wu Q, Gheiratmand L, Comartin D, Tkach JM, Cheung SWT, et al. (2015). A dynamic protein interaction landscape of the human centrosome-cilium interface. Cell $163,1484-1499$.

He K, Ma X, Xu T, Li Y, Hodge A, Zhang Q, Torline J, Huang Y, Zhao J, Ling $K$, et al. (2018). Axoneme polyglutamylation regulated by Joubert syndrome protein ARL13B controls ciliary targeting of signaling molecules. Nat Commun 9, 3310

Hong S-R, Wang C-L, Huang Y-S, Chang Y-C, Chang Y-C, Pusapati GV, Lin C-Y, Hsu N, Cheng H-C, Chiang Y-C, et al. (2018). Spatiotemporal manipulation of ciliary glutamylation reveals its roles in intraciliary trafficking and Hedgehog signaling. Nat Commun 9, 1732.

Hotulainen P, Lappalainen P (2006). Stress fibers are generated by two distinct actin assembly mechanisms in motile cells. J Cell Biol 173, 383-394.

Howe LR, Brown AMC (2004). Wnt signaling and breast cancer. Cancer Biol Ther 3, 36-41.

Inaba H, Goto H, Kasahara K, Kumamoto K, Yonemura S, Inoko A, Yamano S, Wanibuchi H, He D, Goshima N, et al. (2016). Ndel1 suppresses ciliogenesis in proliferating cells by regulating the trichoplein-Aurora $\mathrm{A}$ pathway. J Cell Biol 212, 409-423.

Khan NA, Willemarck N, Talebi A, Marchand A, Binda MM, Dehairs J, Rueda-Rincon N, Daniels VW, Bagadi M, Thimiri Govinda Raj DB, et al. (2016). Identification of drugs that restore primary cilium expression in cancer cells. Oncotarget 7, 9975-9992.

Kim J, Jo H, Hong H, Kim MH, Kim JM, Lee J-K, Heo WDo, Kim J (2015a). Actin remodelling factors control ciliogenesis by regulating YAP/TAZ activity and vesicle trafficking. Nat Commun 6, 6781.

Kim S, Lee K, Choi J-H, Ringstad N, Dynlacht BD (2015b). Nek2 activation of Kif24 ensures cilium disassembly during the cell cycle. Nat Commun 6, 8087.

Kim J, Lee JE, Heynen-Genel S, Suyama E, Ono K, Lee K, Ideker T, Aza-Blanc P, Gleeson JG (2010). Functional genomic screen for modulators of ciliogenesis and cilium length. Nature 464, 1048-1051.

Kim SH, Turnbull J, Guimond S (2011). Extracellular matrix and cell signalling: The dynamic cooperation of integrin, proteoglycan and growth factor receptor. J Endocrinol 209, 21307119.

Lehmann BD, Bauer JA, Chen X, Sanders ME, Chakravarthy AB, Shyr Y, Pietenpol JA (2011). Identification of human triple-negative breast cancer subtypes and preclinical models for selection of targeted therapies. J Clin Invest 121, 2750-2767.

Li H, Deng Y, Sun K, Yang H, Liu J, Wang M, Zhang Z, Lin J, Wu C, Wei Z, et al. (2017). Structural basis of kindlin-mediated integrin recognition and activation. Proc Natl Acad Sci USA 114, 9349-9354.

Majumder S, Fisk HA (2013). VDAC3 and Mps1 negatively regulate ciliogenesis. Cell Cycle 12, 849-858.

Mans DA, Voest EE, Giles RH (2008). All along the watchtower: Is the cilium a tumor suppressor organelle? Biochim Biophys Acta Rev Cancer 1786, 114-125.

Marignani PA, Carpenter CL (2001). Vav2 is required for cell spreading. J Cell Biol 154, 11448999

McDermott KM, Liu BY, TIsty TD, Pazour GJ (2010). Primary cilia regulate branching morphogenesis during mammary gland development. Curr Biol 20, 731-737. 
McGlashan SR, Jensen CG, Poole CA (2006). Localization of extracellular matrix receptors on the chondrocyte primary cilium. J Histochem Cytochem 54, 1005-1014.

Menzl I, Lebeau L, Pandey R, Hassounah NB, Li FW, Nagle R, Weihs K, McDermott KM (2014). Loss of primary cilia occurs early in breast cancer development. Cilia 3, 7.

Miki H, Yamaguchi H, Suetsugu S, Takenawa T (2000). IRSp53 is an essential intermediate between Rac and WAVE in the regulation of membrane ruffling. Nature 408, 732-735.

Nandadasa S, Kraft CM, Wang LW, O'Donnell A, Patel R, Gee HY, Grobe K, Cox TC, Hildebrandt F, Apte SS (2019). Secreted metalloproteases ADAMTS9 and ADAMTS20 have a non-canonical role in ciliary vesicle growth during ciliogenesis. Nat Commun 10, 953.

Nardone G, Oliver-De La Cruz J, Vrbsky J, Martini C, Pribyl J, Skládal P, Pešl M, Caluori G, Pagliari S, Martino F, et al. (2017). YAP regulates cell mechanics by controlling focal adhesion assembly. Nat Commun 8, 28504269.

O'Toole SA, Machalek DA, Shearer RF, Millar EKA, Nair R, Schofield P, McLeod D, Cooper CL, McNeil CM, McFarland A, et al. (2011). Hedgehog overexpression is associated with stromal interactions and predicts for poor outcome in breast cancer. Cancer Res 71, 4002-4014.

Pellegrin S, Mellor H (2007). Actin stress fibres. J Cell Sci 120, $3491-$ 3499.

Pitaval A, Senger F, Letort G, Gidrol X, Guyon L, Sillibourne J, Théry M (2017). Microtubule stabilization drives 3D centrosome migration to initiate primary ciliogenesis. J Cell Biol 216, 3713-3728.

Praetorius HA, Praetorius J, Nielsen S, Frokiaer J, Spring KR (2004). Beta1integrins in the primary cilium of MDCK cells potentiate fibronectininduced Ca2+ signaling. Am J Physiol Renal Physiol 287, F969-F978.

Radisky ES, Radisky DC (2015). Matrix metalloproteinases as drivers and therapeutic targets in breast cancer. Front Biosci 20, 4364.

Rak J, Foster K, Potrzebowska K, Talkhoncheh MS, Miharada N, Komorowska K, Torngren T, Kvist A, Borg Å, Svensson L, et al. (2017). Cytohesin 1 regulates homing and engraftment of human hematopoietic stem and progenitor cells. Blood 129, 950-958.

Rangel L, Bernabé-Rubio M, Fernández-Barrera J, Casares-Arias J, Millán J, Alonso MA, Correas I (2019). Caveolin-1 $\alpha$ regulates primary cilium length by controlling RhoA GTPase activity. Sci Rep 9, 1116.

Sánchez I, Dynlacht BD (2016). Cilium assembly and disassembly. Nat Cell Biol 18, 711-717

Sánchez A, Urrego D, Pardo LA (2016). Cyclic expression of the voltagegated potassium channel KV10.1 promotes disassembly of the primary cilium. EMBO Rep 17, 708-723.

Sarkisian MR, Semple-Rowland SL (2019). Emerging roles of primary cilia in glioma. Front Cell Neurosci 13, 30842728.

Schnelzer A, Prechtel D, Knaus U, Dehne K, Gerhard M, Graeff H, Harbeck N, Schmitt M, Lengyel E (2000). Rac1 in human breast cancer: overexpression, mutation analysis, and characterization of a new isoform, Rac1b. Oncogene 19, 3013-3020.

Schvartz I, Seger D, Shaltiel S (1999). Vitronectin. Int J Biochem Cell Biol 31, 539-544.
Seeger-Nukpezah T, Golemis EA (2012). The extracellular matrix and ciliary signaling. Curr Opin Cell Biol 24, 652-661.

Sorlie T, Perou CM, Tibshirani R, Aas T, Geisler S, Johnsen H, Hastie T, Eisen MB, van de Rijn M, Jeffrey SS, et al. (2001). Gene expression patterns of breast carcinomas distinguish tumor subclasses with clinical implications. Proc Natl Acad Sci 98, 10869-10874.

Sossey-Alaoui K, Pluskota E, Bialkowska K, Szpak D, Parker Y, Morrison CD, Lindner DJ, Schiemann WP, Plow EF (2017). Kindlin-2 regulates the growth of breast cancer tumors by activating CSF-1-mediated macrophage infiltration. Cancer Res 77, 5129-5141.

Tanos BE, Yang H-J, Soni R, Wang W-J, Macaluso FP, Asara JM, Tsou M-FB (2013). Centriole distal appendages promote membrane docking, leading to cilia initiation. Genes Dev 27, 163-168.

Théry M, Pépin A, Dressaire E, Chen Y, Bornens M (2006). Cell distribution of stress fibres in response to the geometry of the adhesive environment. Cell Motil Cytoskeleton 63, 341-355.

Toomer KA, Yu M, Fulmer D, Guo L, Moore KS, Moore R, Drayton KD, Glover J, Peterson N, Ramos-Ortiz S, et al. (2019). Primary cilia defects causing mitral valve prolapse. Sci Transl Med 11, eaax0290.

Wang L, Dynlacht BD (2018). The regulation of cilium assembly and disassembly in development and disease. Development 145, dev151407.

Wheway G, Schmidts M, Mans DA, Szymanska K, Nguyen T-MT, Racher H, Phelps IG, Toedt G, Kennedy J, Wunderlich KA, et al. (2015). An siRNAbased functional genomics screen for the identification of regulators of ciliogenesis and ciliopathy genes. Nat Cell Biol 17, 1074-1087.

Xu Q, Liu W, Liu X, Otkur W, Hayashi T, Yamato M, Fujisaki H, Hattori S, Tashiro S, Ikejima T (2018). Type I collagen promotes primary cilia growth through down-regulating HDAC6-mediated autophagy in confluent mouse embryo fibroblast 3T3-L1 cells. J Biosci Bioeng 125, 8-14.

Yamaji S, Suzuki A, Kanamori H, Mishima W, Yoshimi R, Takasaki H, Takabayashi M, Fujimaki K, Fujisawa S, Ohno S, et al. (2004). Affixin interacts with $\alpha$ actinin and mediates integrin signaling for reorganization of F-actin induced by initial cell-substrate interaction. J Cell Biol 165, 539-551.

Yan B, Calderwood DA, Yaspan B, Ginsberg MH (2001). Calpain cleavage promotes talin binding to the beta 3 integrin cytoplasmic domain. J Biol Chem 276, 28164-28170.

Yuan K, Frolova N, Xie Y, Wang D, Cook L, Kwon YJ, Steg AD, Serra R, Frost AR (2010). Primary cilia are decreased in breast cancer: Analysis of a collection of human breast cancer cell lines and tissues. J Histochem Cytochem 58, 857-870.

Zhang XD (2011). Illustration of SSMD, z Score, SSMD*, $z^{*}$ score, and t statistic for hit selection in RNAi high-throughput screens. J Biomol Screen $16,775-785$.

Zhang X, Jiang G, Cai Y, Monkley SJ, Critchley DR, Sheetz MP (2008). Talin depletion reveals independence of initial cell spreading from integrin activation and traction. Nat Cell Biol 10, 1062-1068.

Zhao B, Wei X, Li W, Udan RS, Yang Q, Kim J, Xie J, Ikenoue T, Yu J, Li L, et al. (2007). Inactivation of YAP oncoprotein by the Hippo pathway is involved in cell contact inhibition and tissue growth control. Genes Dev 21, 17974916. 